\title{
Numerical studies on the electro-optic detection of femtosecond electron bunches
}

\author{
S. Casalbuoni, ${ }^{1}$ H. Schlarb, ${ }^{2}$ B. Schmidt, ${ }^{2}$ P. Schmüser, ${ }^{2,3}$ B. Steffen, ${ }^{2, *}$ and A. Winter ${ }^{3}$ \\ ${ }^{1}$ Institute for Synchrotron Radiation, Research Center Karlsruhe, P.O. Box 3640, D-76021 Karlsruhe, Germany \\ ${ }^{2}$ Deutsches Elektronen-Synchrotron DESY, Notkestrasse 85, 22607 Hamburg, Germany \\ ${ }^{3}$ Institut für Experimentalphysik, Universität Hamburg, Luruper Chaussee 149, 22607 Hamburg, Germany
}

(Received 6 February 2008; published 9 July 2008)

\begin{abstract}
The electro-optic (EO) effect is a powerful diagnostic tool for determining the time profile of ultrashort relativistic electron bunches. When a relativistic bunch passes within a few $\mathrm{mm}$ of an electro-optic crystal, its transient electric field is equivalent to a half-cycle $\mathrm{THz}$ pulse passing through the crystal. The induced birefringence can be detected with polarized femtosecond laser pulses. A simulation code has been written in order to understand the faithfulness and the limitations of electron bunch shape reconstruction by EO sampling. The THz pulse and the laser pulse are propagated as wave packets through the EO crystal. Alternatively, the response function method is applied. Using experimental data on the material properties of zinc telluride ( $\mathrm{ZnTe}$ ) and gallium phosphide $(\mathrm{GaP})$, the effects of velocity mismatch, pulse shape distortion, and signal broadening are explicitly taken into account. The simulations show that the most severe limitation on the time resolution is given by the transverse-optical (TO) lattice oscillation in the EO crystal. The lowest TO frequency is $5.3 \mathrm{THz}$ in $\mathrm{ZnTe}$ and $11 \mathrm{THz}$ in GaP. Only the Fourier components below the TO resonance are usable for the bunch shape reconstruction. This implies that the shortest rms bunch length which can be resolved with moderate distortion amounts to $\sigma \approx 90 \mathrm{fs}$ in ZnTe and $\sigma \approx$ $50 \mathrm{fs}$ in GaP. The influence of the crystal thickness on the amplitude and width of the EO signal is studied. The optimum thickness is in the range from 100 to $300 \mu \mathrm{m}$ for ZnTe and from 50 to $100 \mu \mathrm{m}$ for GaP.

DOI: 10.1103/PhysRevSTAB.11.072802

PACS numbers: 41.60.Cr, 42.65.Re, 41.85.Ew, 42.81.Gs
\end{abstract}

\section{INTRODUCTION}

The electro-optic (EO) method has been successfully applied at several accelerators to study the time structure of ultrashort electron bunches [1-3]. The best time resolution has been achieved in a recent experiment [4] at the ultraviolet and soft $\mathrm{x}$-ray free-electron laser FLASH (Free-electron LAser in Hamburg) at an electron energy of $450 \mathrm{MeV}$ and a free-electron laser (FEL) wavelength of $30 \mathrm{~nm}$. The FEL is based on the principle of self-amplified spontaneous emission (SASE) which opens the way to powerful lasers in the $\mathrm{x}$-ray regime and requires electron bunches of extremely high local charge density to achieve laser saturation in the $27 \mathrm{~m}$ long undulator magnet. Electron bunches with a charge of $0.5 \mathrm{nC}$ and an rms time duration of $\sigma=5 \mathrm{ps}$ are generated in a radio frequency photocathode, accelerated to relativistic energies and then longitudinally compressed by 2 orders of magnitude in a two-stage bunch compression scheme. The compressed bunches are characterized by a short leading spike with an rms length of less than 50 fs followed by a tail that is several ps long. Precise measurements of the temporal profile of the compressed electron bunches are essential for the optimization of the accelerator and a proper understanding of the bunch compression mechanism including subtle effects such as coherent synchrotron radiation and space charge forces. For this purpose an electro-optic detection system [4] has been installed at FLASH with an EO crystal placed inside the electron-beam vacuum

*Present address: Paul Scherrer Institut, Villigen, Switzerland. chamber at a few millimeter distance from the beam. A major concern are pulse shape distortions in the electrooptic detection process which are known to happen for ultrashort THz pulses, see, e.g., Ref. [5]. Since quantitative calculations on the EO detection of femtosecond electron bunches were not available to us we have carried out detailed numerical studies on the electro-optic effect in gallium phosphide $(\mathrm{GaP})$ and zinc telluride $(\mathrm{ZnTe})$ with the aim of assessing the faithfulness and the limitations of EO bunch shape reconstruction. In the model calculations we restrict ourselves to the simple method of electro-optic sampling (EOS), where a narrow laser pulse is scanned in small time steps across the THz pulse. EOS yields the best possible resolution if timing jitter between the laser pulses and the electron bunches is negligible. In practical accelerators time jitter is significant, and one has to resort to single-shot techniques such as spectral, temporal, or spatial decoding [1-4] if one wants to resolve ultrashort electron bunches. These more advanced EO techniques can at best achieve the time resolution of a jitter-free EOS experiment and will not be discussed here. Our simulation results are nevertheless relevant for single-shot EO measurements if the additional resolution limitations are properly accounted for.

When a relativistic bunch passes within a few $\mathrm{mm}$ of an electro-optic crystal, its transient electric field is equivalent to a half-cycle $\mathrm{THz}$ pulse impinging on the crystal. The temporal profile of this equivalent half-cycle $\mathrm{THz}$ pulse provides a faithful image of the longitudinal charge distribution inside the electron bunch if the electrons are highly relativistic (typically for a Lorentz factor $\gamma>1000$ ). Using 
the available experimental data on the complex refractive index $n(\omega)+i \kappa(\omega)$ in the THz regime, we calculate the transmission of this ultrashort electric field pulse from the accelerator vacuum into the EO crystal and its propagation in the material. The frequency dependencies of the complex refractive index $n(\omega)+i \kappa(\omega)$ and the EO coefficient $r_{41}(\omega)$ lead to pulse broadening and shape distortions which may become severe for very short bunches. In particular high-frequency oscillations develop in the EO crystal.

Ideally, the THz pulse and the laser pulse should move with the same speed through the EO crystal to obtain the best time resolution and a maximum signal in the EO system. The detrimental effects of group and phase velocity mismatch are investigated in detail. The main limitation for the shortest resolvable time structure is given by the excitation of transverse-optical (TO) lattice vibrations. The lowest TO frequency amounts to $5.3 \mathrm{THz}$ for $\mathrm{ZnTe}$ and $11 \mathrm{THz}$ for GaP. Near a resonance the refractive index is rapidly changing and severe velocity mismatch occurs. We will show that $\mathrm{GaP}$ is better suited to measure very short pulses owing to its higher TO frequency. The disadvantage is that the electro-optic coefficient of $\mathrm{GaP}$ is significantly smaller than that of ZnTe.

The paper is organized as follows. The theoretical background of the EO effect is presented in Sec. II which has been adapted from a previous report [6], see also Refs. [7,8]. In Sec. III we collect the available experimental data on the relevant material properties of $\mathrm{GaP}$ and ZnTe. The numerical simulation is explained in Sec. IV. First a physically intuitive approach is followed in which the THz pulse and the laser pulse are propagated as wave packets through the crystal. Then the response function method is explained. Both approaches lead to identical EO signals if the laser pulse retains its shape while propagating through the EO crystal. The quality of bunch shape reconstruction and the distortions arising for extremely short bunches are discussed in Sec. V.

\section{ELECTRO-OPTIC EFFECTS IN ANISOTROPIC CRYSTALS}

For a homogeneous medium the electric displacement vector is given by

$$
\boldsymbol{D}=\varepsilon_{0} \varepsilon \boldsymbol{E},
$$

where the (relative) dielectric permittivity $\varepsilon$ is a scalar quantity (independent of direction). In a crystal the polarization may depend on the direction of the electric field with respect to the crystallographic axes. In this case the permittivity is a symmetric tensor $\hat{\varepsilon}$, and $\boldsymbol{D}$ is in general not parallel to the electric field. It is always possible to carry out a principal-axis transformation to an orthogonal coordinate system in which $\boldsymbol{E}$ and $\boldsymbol{D}$ are related by a diagonal matrix:

$$
\left(\begin{array}{c}
D_{1} \\
D_{2} \\
D_{3}
\end{array}\right)=\varepsilon_{0}\left(\begin{array}{ccc}
\varepsilon_{1} & 0 & 0 \\
0 & \varepsilon_{2} & 0 \\
0 & 0 & \varepsilon_{3}
\end{array}\right) \cdot\left(\begin{array}{c}
E_{1} \\
E_{2} \\
E_{3}
\end{array}\right) .
$$

If the $\varepsilon_{i}$ are not all identical the crystal exhibits birefringence (double refraction).

The energy density of the electric field is $w_{e}=\frac{1}{2} \boldsymbol{E} \cdot \boldsymbol{D}$. Using Eq. (2) we can show that the surfaces of constant energy density are ellipsoids in the $\boldsymbol{D}$ space:

$$
2 \varepsilon_{0} w_{e}=\boldsymbol{D} \cdot \hat{\varepsilon}^{-1} \cdot \boldsymbol{D}=\frac{D_{1}^{2}}{\varepsilon_{1}}+\frac{D_{2}^{2}}{\varepsilon_{2}}+\frac{D_{3}^{2}}{\varepsilon_{3}} .
$$

Defining a dimensionless vector along the direction of $\boldsymbol{D}$ by $\boldsymbol{u}=\boldsymbol{D} / \sqrt{2 \varepsilon_{0} w_{e}}$, we get the equation of the refractive index ellipsoid

$$
\frac{u_{1}^{2}}{n_{1}^{2}}+\frac{u_{2}^{2}}{n_{2}^{2}}+\frac{u_{3}^{2}}{n_{3}^{2}}=1
$$

where $n_{i}=\sqrt{\varepsilon_{i}}$ for a nonmagnetic material. Defining the impermeability tensor by

$$
\hat{\boldsymbol{\eta}}=\hat{\varepsilon}^{-1},
$$

the ellipsoid equation can be written as

$$
\boldsymbol{u} \cdot \hat{\boldsymbol{\eta}} \cdot \boldsymbol{u}=1 .
$$

\section{A. Electro-optic effect in zinc telluride and gallium phosphide}

ZnTe and GaP have a cubic crystal lattice and are optically isotropic at vanishing electric field (i.e. $\varepsilon_{1}=$ $\varepsilon_{2}=\varepsilon_{3} \equiv \varepsilon$ ), which means that the impermeability tensor can be replaced by the scalar quantity $\varepsilon^{-1}$, multiplied with the unit matrix $\boldsymbol{I}$. In the presence of an electric field the impermeability tensor becomes [7]

$$
\hat{\boldsymbol{\eta}}(\boldsymbol{E})=\varepsilon^{-1} \boldsymbol{I}+\boldsymbol{r} \cdot \boldsymbol{E} .
$$

The second term describes the Pockels effect, and $\boldsymbol{r}$ is the EO tensor. The Kerr effect which is quadratic in the electric field is neglected here. The ellipsoid equation is now

$$
\boldsymbol{u} \cdot \hat{\boldsymbol{\eta}}(\boldsymbol{E}) \cdot \boldsymbol{u}=\sum_{i, j=1,2,3}\left(\varepsilon^{-1} \delta_{i j}+\sum_{k=1,2,3} r_{i j k} E_{k}\right) u_{i} u_{j}=1 .
$$

TABLE I. Convention for replacing the first two indices $i, j$ of the tensor $\boldsymbol{r}$ by a single index in case of a symmetric impermeability tensor $\left(r_{i j k}=r_{j i k}\right)$.

\begin{tabular}{ll}
\hline \hline$(1,1) \rightarrow 1$ & $r_{11 k} \rightarrow r_{1 k}$ \\
$(2,2) \rightarrow 2$ & $r_{22 k} \rightarrow r_{2 k}$ \\
$(3,3) \rightarrow 3$ & $r_{33 k} \rightarrow r_{3 k}$ \\
$(2,3) \rightarrow 4$ & $r_{23 k}=r_{32 k} \rightarrow r_{4 k}$ \\
$(1,3) \rightarrow 5$ & $r_{13 k}=r_{31 k} \rightarrow r_{5 k}$ \\
$(1,2) \rightarrow 6$ & $r_{12 k}=r_{21 k} \rightarrow r_{6 k}$ \\
\hline \hline
\end{tabular}


The tensor $\hat{\boldsymbol{\eta}}$ is symmetric, hence $r_{i j k}=r_{j i k}$. It is convention to replace the first two indices $i, j$ of the tensor $\boldsymbol{r}$ by a single index, see Table I.

$\mathrm{GaP}$ and ZnTe crystallize in the zinc blende structure (two face-centered cubic lattices which are shifted against each other by one quarter of the spatial diagonal). Owing to the high degree of symmetry, the GaP (ZnTe) crystal is optically isotropic at vanishing electric fields, i.e. $n_{1}=$ $n_{2}=n_{3}=n_{0}$, and the tensor $\boldsymbol{r}$ is characterized by a single quantity $r_{41}=r_{52}=r_{63}$. Hence, the equation of the refractive index ellipsoid assumes the form

$\frac{1}{n_{0}^{2}}\left(u_{1}^{2}+u_{2}^{2}+u_{3}^{2}\right)+2 r_{41}\left(E_{1} u_{2} u_{3}+E_{2} u_{3} u_{1}+E_{3} u_{1} u_{2}\right)=1$.

To obtain the modified refractive indices one has to perform a principal-axis transformation.

\section{B. Determination of the main refractive indices}

The GaP or ZnTe crystals used in EO experiments are cut in the (110) plane as shown in Fig. 1. The electric field pulse and the laser pulse impinge perpendicular to this plane along the direction $[-1,-1,0]$, their electric vectors lie therefore in the (110) plane. We define a twodimensional coordinate system $(X, Y)$ in this plane where $X$ points along the $[-1,1,0]$ direction and $Y$ along the $[0,0,1]$ direction.

Let the electric vector $\boldsymbol{E}$ of the $\mathrm{THz}$ pulse enclose an angle $\alpha$ with the $X$ axis (the $[-1,1,0]$ axis of the EO crystal). Its components in the base system of the cubic crystal lattice are

$$
\boldsymbol{E}=E\left(\begin{array}{c}
-\cos \alpha / \sqrt{2} \\
\cos \alpha / \sqrt{2} \\
\sin \alpha
\end{array}\right)
$$

Equation (9) reads in this special case

$$
\boldsymbol{u} \cdot \hat{\boldsymbol{\eta}}(\boldsymbol{E}) \cdot \boldsymbol{u}=1
$$
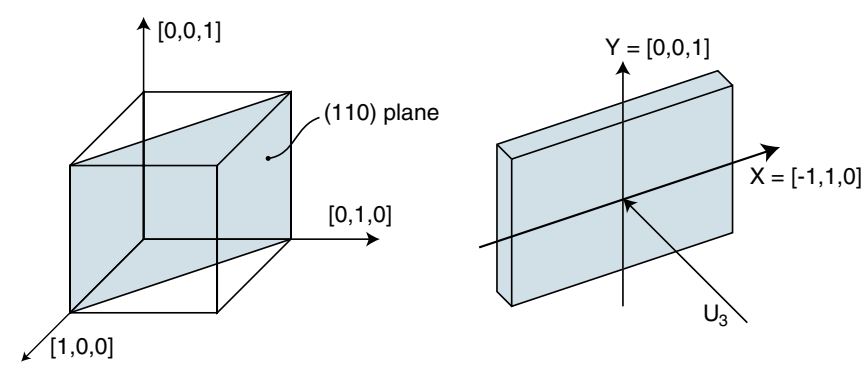

FIG. 1. (Color) Left: The (110) plane in the cubic gallium phosphide (zinc telluride) crystal. Right: The coordinate system $(X, Y)$ in the (110) plane. The $\mathrm{THz}$ pulse and the laser pulse impinge along the direction of the normal to this plane (unit vector $\boldsymbol{U}_{3}$ ). with the field-dependent impermeability tensor

$$
\begin{aligned}
\hat{\boldsymbol{\eta}}(\boldsymbol{E})= & \frac{1}{n_{0}^{2}}\left(\begin{array}{lll}
1 & 0 & 0 \\
0 & 1 & 0 \\
0 & 0 & 1
\end{array}\right) \\
& +r_{41} E\left(\begin{array}{ccc}
0 & \sin \alpha & \cos \alpha / \sqrt{2} \\
\sin \alpha & 0 & -\cos \alpha / \sqrt{2} \\
\cos \alpha / \sqrt{2} & -\cos \alpha / \sqrt{2} & 0
\end{array}\right) .
\end{aligned}
$$

The eigenvalues of the tensor are for $0 \leq \alpha<\pi / 2$

$$
\begin{aligned}
\lambda_{1,2} & =\frac{1}{n_{0}^{2}}-\frac{r_{41} E}{2}\left(\sin \alpha \pm \sqrt{1+3 \cos ^{2} \alpha}\right) \\
\lambda_{3} & =\frac{1}{n_{0}^{2}}+r_{41} E \sin \alpha,
\end{aligned}
$$

and the normalized eigenvectors are

$\boldsymbol{U}_{1}=\frac{1}{2} \sqrt{1+\frac{\sin \alpha}{\sqrt{1+3 \cos ^{2} \alpha}}}\left(\begin{array}{c}-1 \\ 1 \\ \frac{2 \sqrt{2} \cos \alpha}{\sqrt{1+3 \cos ^{2} \alpha}+\sin \alpha}\end{array}\right)$

$\boldsymbol{U}_{2}=\frac{1}{2} \sqrt{1-\frac{\sin \alpha}{\sqrt{1+3 \cos ^{2} \alpha}}}\left(\begin{array}{c}1 \\ -1 \\ \frac{2 \sqrt{2} \cos \alpha}{\sqrt{1+3 \cos ^{2} \alpha}-\sin \alpha}\end{array}\right)$

$\boldsymbol{U}_{3}=\frac{1}{\sqrt{2}}\left(\begin{array}{c}-1 \\ -1 \\ 0\end{array}\right)$.

The principal axes point in the direction of the eigenvectors. The main refractive indices are given by

$$
n_{i}=1 / \sqrt{\lambda_{i}} \text {. }
$$

Considering that $r_{41} E \ll 1 / n_{0}^{2}$ this yields in good approximation

$$
\begin{aligned}
& n_{1}=n_{0}+\frac{n_{0}^{3} r_{41} E}{4}\left(\sin \alpha+\sqrt{1+3 \cos ^{2} \alpha}\right) \\
& n_{2}=n_{0}+\frac{n_{0}^{3} r_{41} E}{4}\left(\sin \alpha-\sqrt{1+3 \cos ^{2} \alpha}\right) \\
& n_{3}=n_{0}-\frac{n_{0}^{3} r_{41} E}{2} \sin \alpha .
\end{aligned}
$$

From Eq. (13) it is obvious that the principal axis $\boldsymbol{U}_{3}$ is perpendicular to the (110) crystal plane. This is also the direction of incidence of the $\mathrm{THz}$ pulse and the titaniumsapphire (Ti:Sa) laser pulse. The vector $\boldsymbol{U}_{1}$ lies in the (110) plane and encloses an angle $\psi$ with the $[-1,1,0]$ axis which can be calculated using the scalar product and the relation $\cos (2 \psi)=2 \cos ^{2} \psi-1$. One gets

$$
\cos 2 \psi=\frac{\sin \alpha}{\sqrt{1+3 \cos ^{2} \alpha}} .
$$

The second principal axis is parallel to the vector $\boldsymbol{U}_{2}$ and 


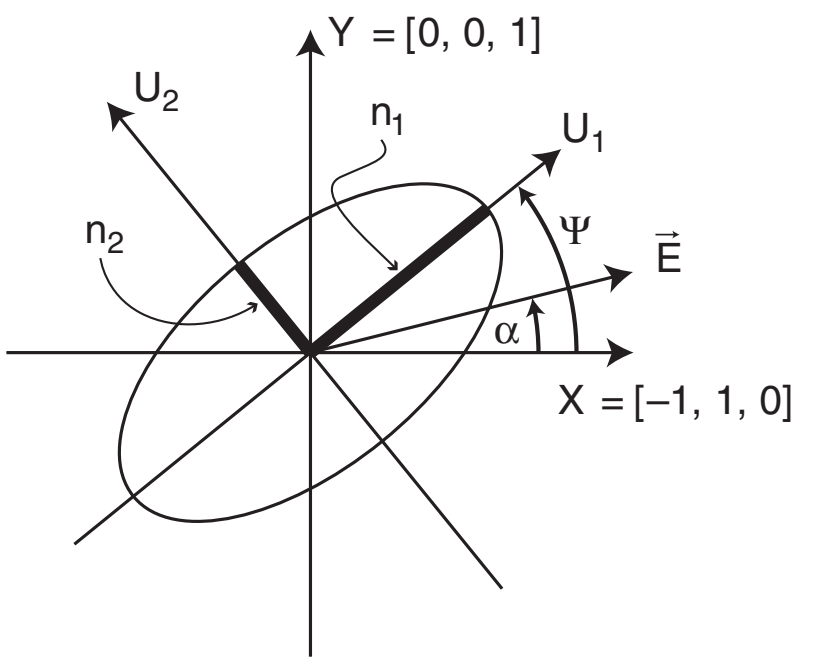

FIG. 2. The refractive index ellipsoid projected onto the (110) plane of the electro-optic crystal. The difference between the refractive indices $n_{1}$ and $n_{2}$ is strongly exaggerated. The electric vector $\boldsymbol{E}$ of the THz pulse encloses an angle $\alpha$ with the $X=$ $[-1,1,0]$ axis of the EO crystal while the angle between the long half axis of the ellipse and the $X$ axis is given by $\psi(\alpha)$. Both the $\mathrm{THz}$ pulse and the laser pulse impinge along the normal to the (110) plane. In the simulations we choose $\alpha=0$ and hence $\psi=$ $\pi / 4$, and assume a linear laser polarization parallel to the $[-1,1,0]$ axis.

perpendicular to $\boldsymbol{U}_{1}$. Note that for $\alpha=\pi / 2$ the principal axis $\boldsymbol{U}_{1}$ points in the $X$ direction, and the axis $\boldsymbol{U}_{2}$ in the $Y$ direction.

The principal refractive indices corresponding to the first two principal axes are $n_{1}$ and $n_{2}$. The refractive index ellipse is shown in Fig. 2.

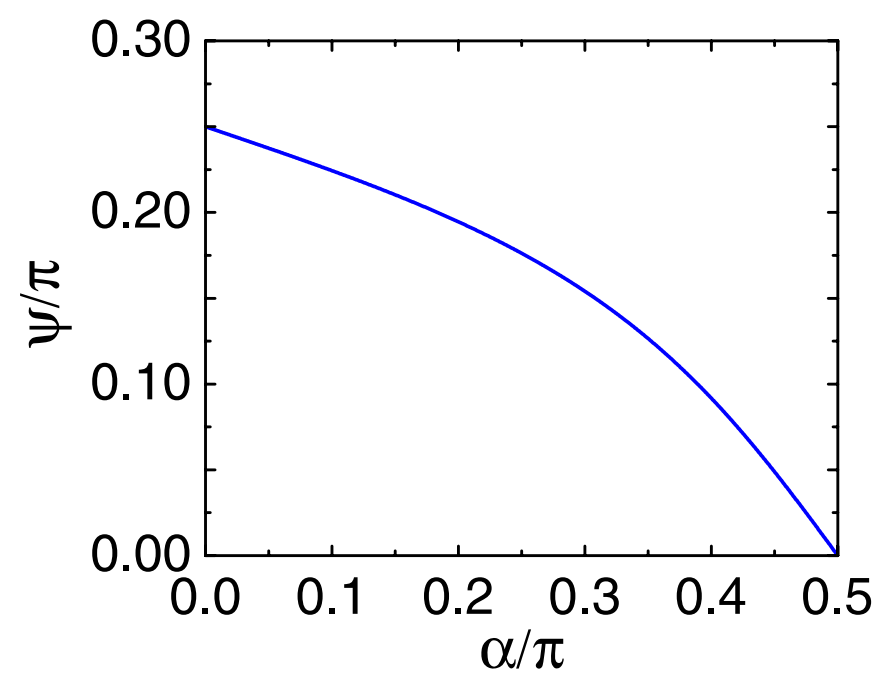

The Ti:Sa laser beam is incident onto the EO crystal along the normal to the (110) plane, its electric vector $\boldsymbol{E}_{\text {las }}$ lies therefore in this plane. In the crystal the two components of $\boldsymbol{E}_{\text {las }}$ along the principal axes $\boldsymbol{U}_{1}$ and $\boldsymbol{U}_{2}$ receive a relative phase shift

$$
\Gamma=\frac{2 \pi\left(n_{1}-n_{2}\right) d}{\lambda_{0}}=\frac{\pi n_{0}^{3} d}{\lambda_{0}} r_{41} E \sqrt{1+3 \cos ^{2} \alpha},
$$

where $\lambda_{0}$ is the wavelength of the laser light in vacuum and $d$ the thickness of the crystal. The rotation angle $\psi$ of the index ellipse in the $(X, Y)$ plane and the relative phase shift $\Gamma$, also called the retardation parameter, are plotted in Fig. 3 as a function of the angle $\alpha$ between the applied electric vector $\boldsymbol{E}$ and the $X$ axis. The geometry leading to the largest electro-optical effect is the following: the $\mathrm{THz}$ pulse is incident perpendicular to the (110) plane and is linearly polarized parallel to the $[-1,1,0]$ axis. Then $\alpha=$ 0 and hence $\psi=\pi / 4$, so the retardation parameter becomes

$$
\Gamma=\frac{2 \pi n_{0}^{3} d}{\lambda_{0}} r_{41} E
$$

In all our simulations, the probe laser pulse is assumed to be incident perpendicular to the (110) plane and linearly polarized parallel to the $[-1,1,0]$ axis. More details on the orientation dependence of terahertz pulse detection in EO crystals are reported in [9].

\section{Principle of signal detection}

Here we give only a short summary of the signal detection scheme, for a detailed treatment we refer to [6]. A high sensitivity can be achieved with a balanced diode detector. For that purpose the laser beam leaving the EO crystal is

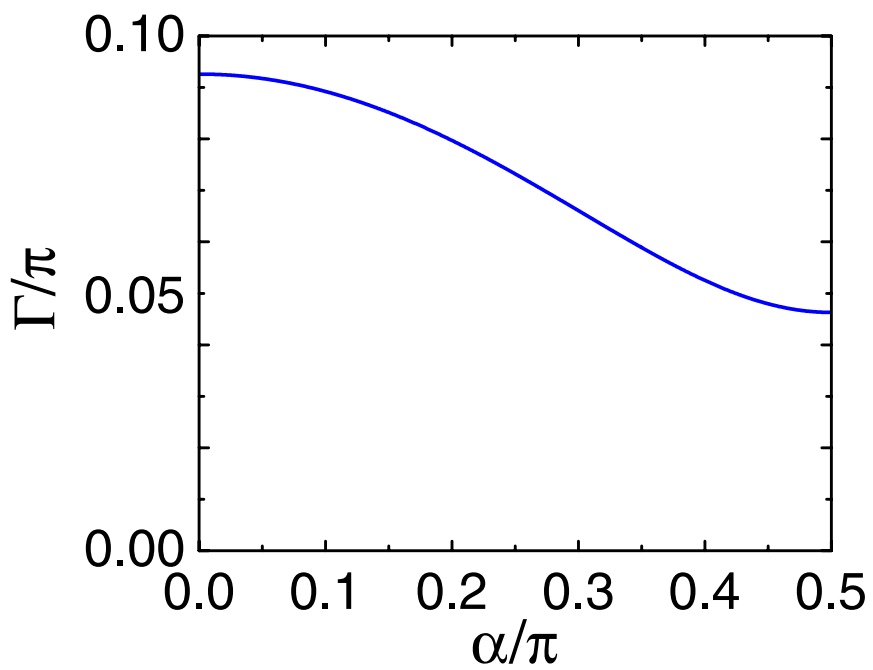

FIG. 3. (Color) Left: The angle $\psi$ between the first principal axis of the refractive index ellipsoid and the $X$ axis as a function of the angle $\alpha$ between the applied electric field vector $\boldsymbol{E}$ and the $X$ axis. Right: The retardation parameter $\Gamma$, i.e., the relative phase shift between the two orthogonal components of the laser field $\boldsymbol{E}_{\text {las }}$, plotted as a function of $\alpha$. The curve is computed for a ZnTe crystal with the following parameters: $d=200 \mu \mathrm{m}, r_{41}=4 \times 10^{-12} \mathrm{~m} / \mathrm{V}, E=2 \times 10^{6} \mathrm{~V} / \mathrm{m}, n_{0}=2.85$, and $\lambda_{0}=800 \mathrm{~nm}$. 


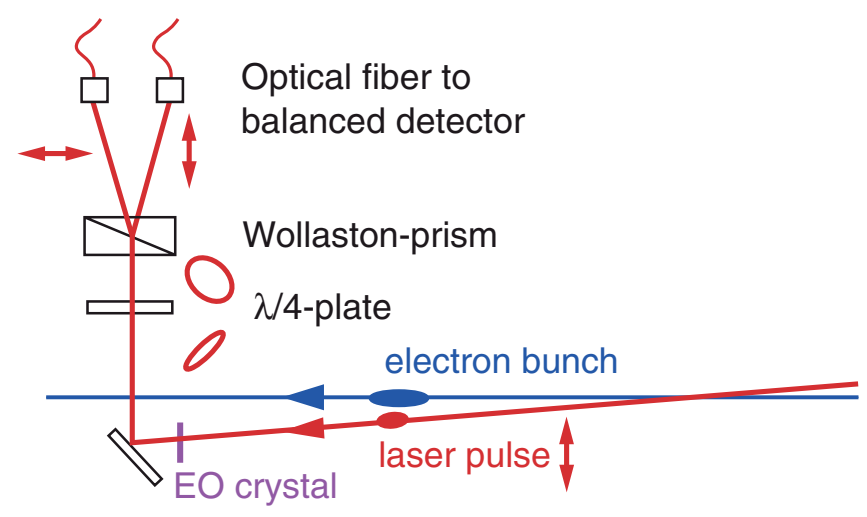

FIG. 4. (Color) Simplified top view of electro-optic signal detection using a quarter wave plate, a Wollaston prism, and a balanced diode detector. The incident laser beam is polarized horizontally. The quarter wave plate is rotated by $45^{\circ}$ with respect to the horizontal plane.

passed through a quarter wave plate whose main axes are oriented at $\pm 45^{\circ}$ with respect to the horizontal direction (i.e. the $X$ axis). A Wollaston prism separates the two polarization components and guides them to the two diodes of a balanced detector. The arrangement is sketched in Fig. 4. If the electric field of the bunch is absent the Ti:Sa laser pulse remains unaffected by the EO crystal and leaves it with horizontal polarization. The quarter wave plate transforms this to circular polarization. The Wollaston prism guides the two orthogonal components of the circular wave to the two diodes which record then of course the same intensity. Hence, the difference signal vanishes. In the presence of an electric field, however, the radiation behind the EO crystal is elliptically polarized leading to an imbalance between the two diode signals. The difference signal is proportional to the sine of the retardation parameter [6]

$$
\left|A_{1}\right|^{2}-\left|A_{2}\right|^{2} \propto \sin (\Gamma)
$$

where $A_{1}$ and $A_{2}$ are the light amplitudes in detector 1 , respectively detector 2 .

\section{MATERIAL PROPERTIES}

\section{A. Refractive index in the visible range}

The index of refraction for visible and infrared light has been measured both for GaP $[10,11]$ and ZnTe $[12,13]$. The optical refractive index of $\mathrm{GaP}$ is described by [14]

$$
n(\lambda)=\sqrt{2.680+\frac{6.40 \lambda^{2}}{\lambda^{2}-0.0903279}},
$$

where $\lambda$ is the photon wavelength in $\mu \mathrm{m}$. A useful parametrization for $\mathrm{ZnTe}$ is [12]

$$
n(\lambda)=\sqrt{4.27+\frac{3.01 \lambda^{2}}{\lambda^{2}-0.142}} .
$$

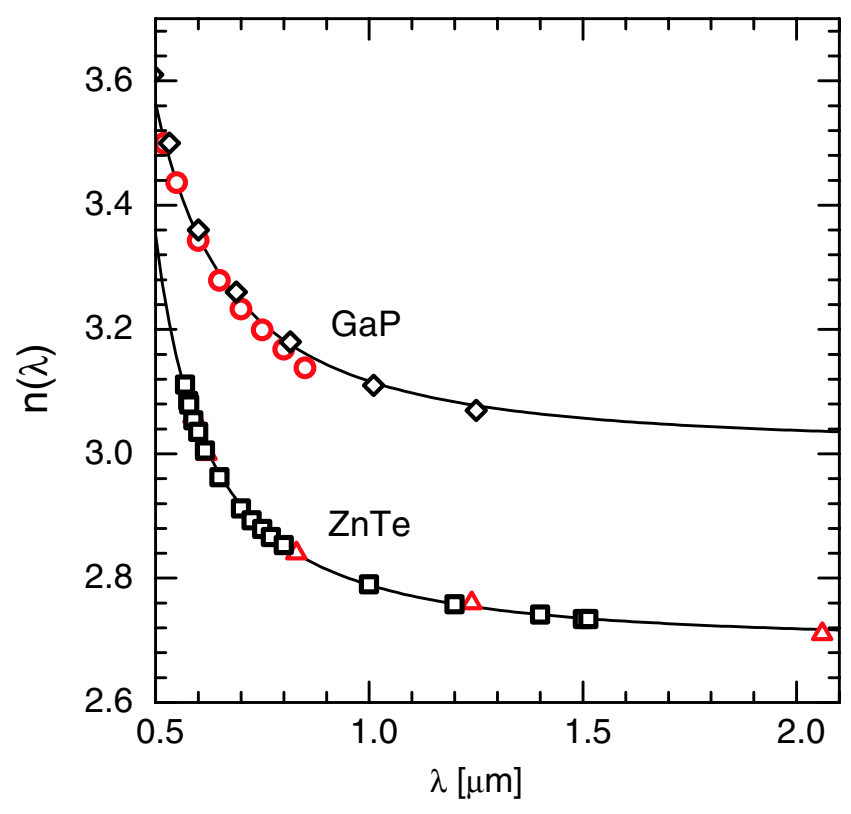

FIG. 5. (Color) The refractive index of GaP and $\mathrm{ZnTe}$ for visible and infrared light. GaP: the experimental data are indicated by circles [10], respectively diamonds [11]; solid curve: fit according to Eq. (19). ZnTe: experimental data are indicated by squares [13], respectively triangles [12]; solid curve: fit according to Eq. (20).

The optical refractive indices of $\mathrm{GaP}$ and $\mathrm{ZnTe}$ are plotted in Fig. 5 as a function of wavelength.

\section{B. Refractive index in the $\mathrm{THz}$ range}

For frequencies far below the optical regime, the complex dielectric function $\varepsilon(\omega)$ of a nonconducting crystal can be written in the form [15]

$$
\varepsilon(\omega)=\varepsilon_{\mathrm{el}}+\sum_{j} \frac{S_{j} \omega_{j}^{2}}{\omega_{j}^{2}-\omega^{2}-i \Lambda_{j} \omega},
$$

where the first term is the contribution from the bound electrons and the second term the contribution from lattice oscillations which are treated as damped harmonic oscillators. The coefficient $\varepsilon_{\mathrm{el}}$ is constant in the $\mathrm{THz}$ frequency range. The sum extends over all lattice oscillations which couple to the electromagnetic field. The quantities $\omega_{j}, \Lambda_{j}$, and $S_{j}$ are the eigenfrequency, damping constant, and oscillator strength of the respective lattice oscillation. For the electro-optic crystals GaP and ZnTe, a good description of $\varepsilon(\omega)$ in the $\mathrm{THz}$ regime is obtained by restricting the sum to the lowest transverse-optical (TO) lattice oscillation:

$$
\varepsilon(\omega)=\varepsilon_{\mathrm{el}}+\frac{S_{0} \omega_{0}^{2}}{\omega_{0}^{2}-\omega^{2}-i \Lambda_{0} \omega} .
$$

The complex index of refraction is given by taking the square root: 

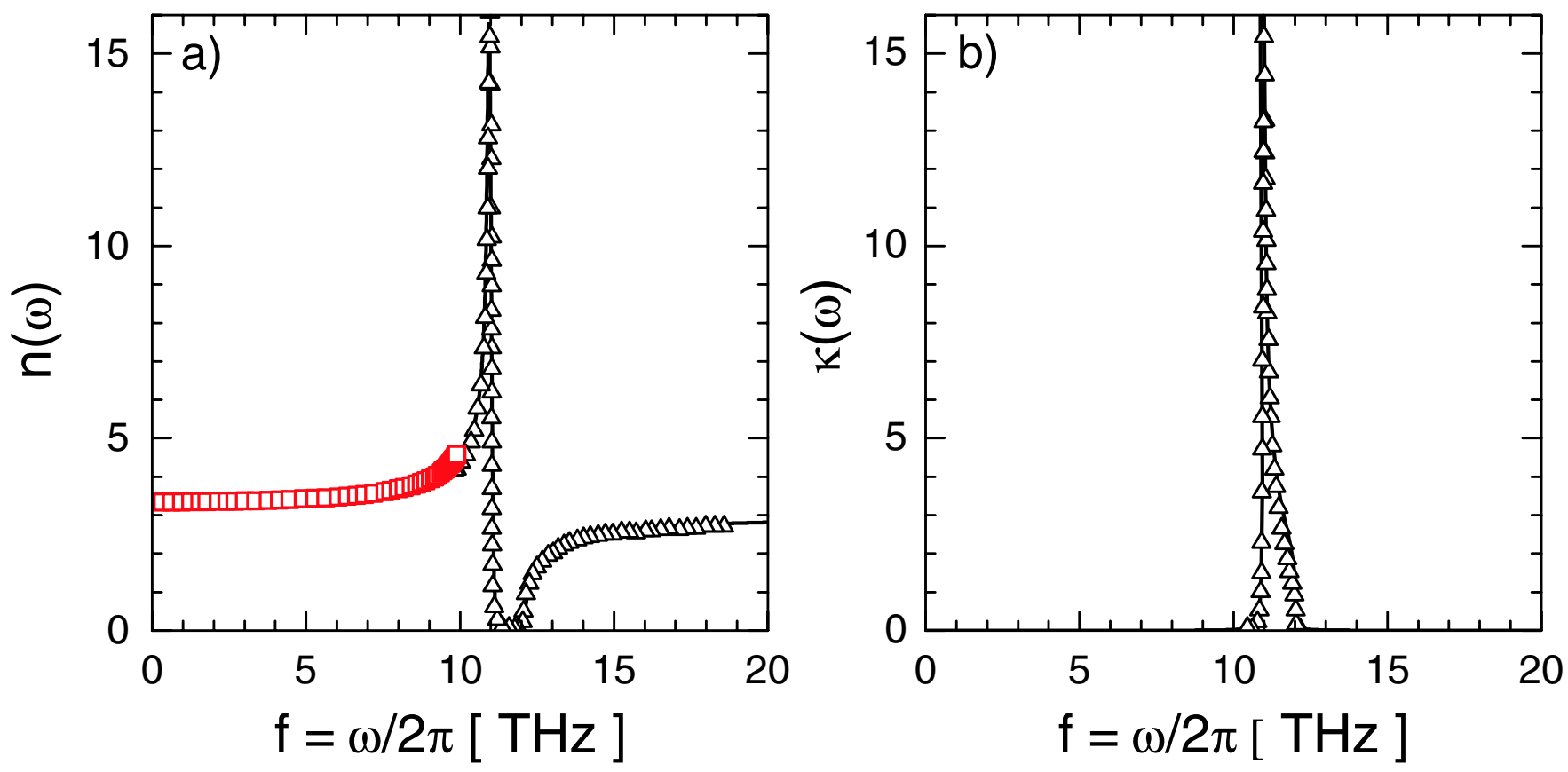

FIG. 6. (Color) (a) Real part $n(\omega)$ and (b) imaginary part $\kappa(\omega)$ of the refractive index of GaP. Here the lowest TO resonance is at $11 \mathrm{THz}$. The triangles $\triangle$ are experimental data taken from Ref. [30], the squares $\square$ from Ref. [23]. The curves are fits of the experimental data using the formulas and the parameters shown in the text.
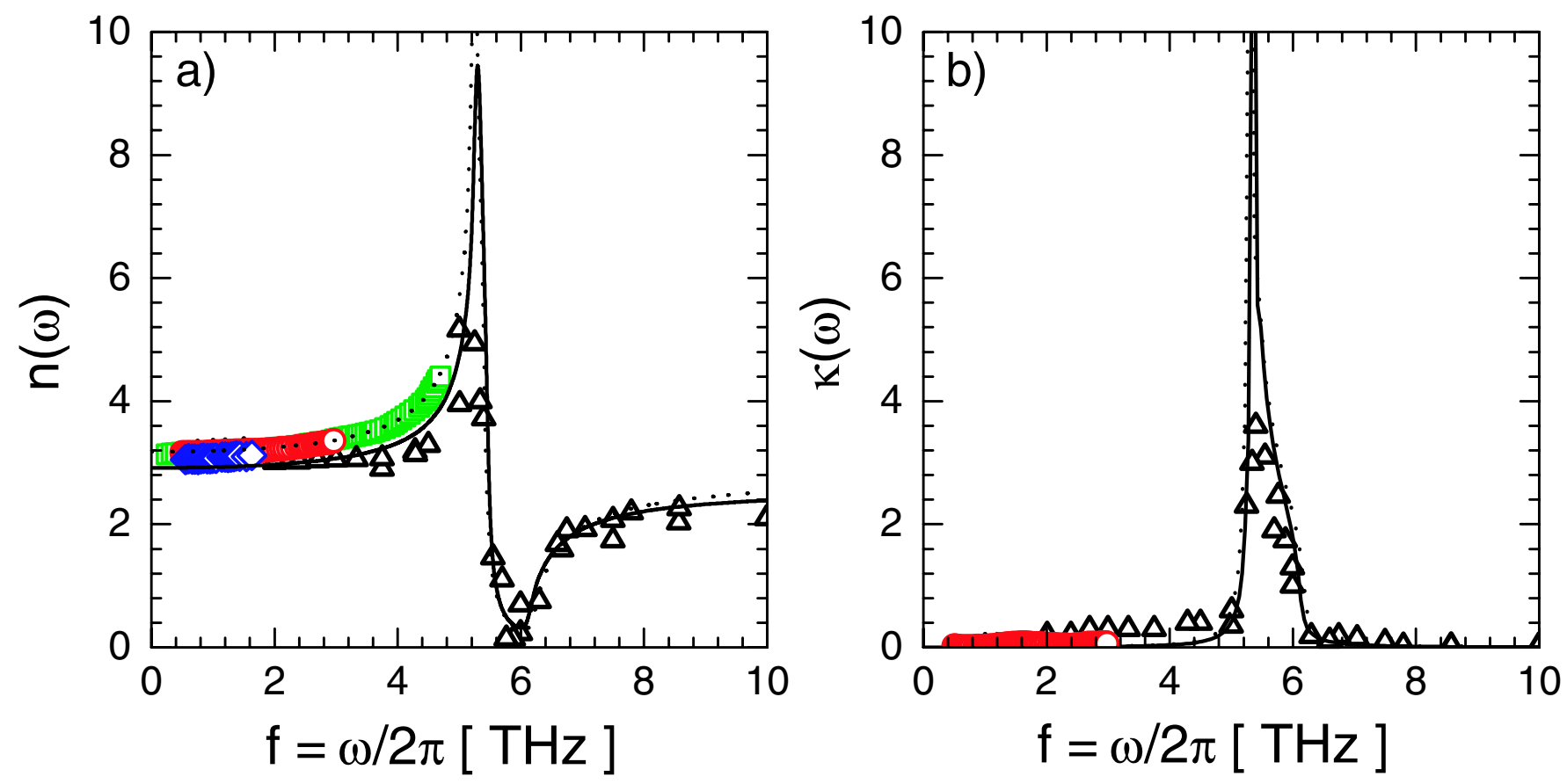

FIG. 7. (Color) (a) Real part $n(\omega)$ and (b) imaginary part $\kappa(\omega)$ of the refractive index of ZnTe in the THz range. The resonance at $5.3 \mathrm{THz}$ is due to the excitation of a transverse-optical (TO) lattice vibration. The square symbols $\square$ are experimental data taken from Ref. [16], the triangles $\triangle$ from Ref. [13], the diamonds $\diamond$ from Ref. [31], and the circles $\bigcirc$ from Ref. [32]. The curves are fits of the experimental data using the formulas and the parameters shown in the text. The dotted line corresponds to the first set of parameters, while the solid line to the second set of parameters. 


$$
n(\omega)+i \kappa(\omega)=\sqrt{\varepsilon(\omega)} .
$$

In Figs. 6 and 7 we plot the published experimental data for $\mathrm{GaP}$, respectively $\mathrm{ZnTe}$, on the real and imaginary part of the refractive index as a function of frequency. The curves are fits of the experimental data using the formulas (22) and (23) with the following parameters for $\mathrm{GaP}$ :

$$
\begin{array}{lll}
\mathrm{GaP}: & \varepsilon_{\mathrm{el}}=8.7, & f_{0}=\omega_{0} /(2 \pi)=10.98 \mathrm{THz}, \\
& S_{0}=1.8, & \Lambda_{0} /(2 \pi)=0.02 \mathrm{THz} .
\end{array}
$$

For $\mathrm{ZnTe}$ we observe a variation up to $25 \%$ between the data from Ref. [16] and those from Ref. [13]. Thus, we have determined two different sets of fit parameters. For Ref. [16] we obtain

$$
\begin{array}{lll}
\text { ZnTe: } & \varepsilon_{\mathrm{el}}=7.4, & f_{0}=\omega_{0} /(2 \pi)=5.3 \mathrm{THz}, \\
& S_{0}=2.7, \quad \Lambda_{0} /(2 \pi)=0.09 \mathrm{THz},
\end{array}
$$

while for Ref. [13]

$$
\begin{array}{lll}
\text { ZnTe: } & \varepsilon_{\mathrm{el}}=6.5, & f_{0}=5.35 \mathrm{THz}, \\
& S_{0}=2.0, & \Lambda_{0} /(2 \pi)=0.09 \mathrm{THz} .
\end{array}
$$

In the following simulations we use the first set of parameters. The second set of parameters yields practically the same results if the THz pulse is longer than $50 \mathrm{fs}(\mathrm{rms})$.

\section{Electro-optic coefficient}

The electro-optic coefficient $r_{41}$ is affected by the same lattice oscillations as the dielectric function. It is therefore suggestive to parametrize it in the form [17]

$$
r_{41}(\omega)=d_{E}\left(1+\frac{C \omega_{0}^{2}}{\omega_{0}^{2}-\omega^{2}-i \Lambda_{0} \omega}\right)
$$

with the same parameters $\omega_{0}$ and $\Lambda_{0}$ as used above. The literature values on the absolute magnitude $\left|r_{41}\right|$ for $\mathrm{GaP}$ are collected in Fig. 8 and compared with the frequency dependence given by Eq. (24) with the parameters $d_{E}=$ $1 \times 10^{-12} \mathrm{~m} / \mathrm{V}$ and $C=-0.53$ [17].

In Fig. 9 we show the rather sparse published data on $\left|r_{41}\right|$ for ZnTe. A very small value of $\left|r_{41}\right|$ at $30 \mathrm{THz}$ was reported in Ref. [18]. It has been omitted from the figure since it is in gross disagreement with the other data [19] at this frequency and the values found in the visible range [20]. A possible reason for this discrepancy might be the low resistivity value of $\rho \approx 10^{3} \Omega \mathrm{m}$ of the sample of Ref. [18] compared to $\rho \approx 4 \times 10^{6} \Omega \mathrm{m}$ in Ref. [20] and $\rho \approx 10^{7} \Omega \mathrm{m}$ in Ref. [19]. The experimental data are far too scarce to derive any frequency dependence of $r_{41}$, but in analogy with $\mathrm{GaP}$ we are justified to assume a frequency dependence according to Eq. (24). Because of the low piezoelectric constant of $\mathrm{ZnTe}[20,21]$, the value of the constant $C$ is expected to be small, so the unknown lowfrequency value of $r_{41}$ should not differ appreciably from the values measured at high frequency. The curve shown in

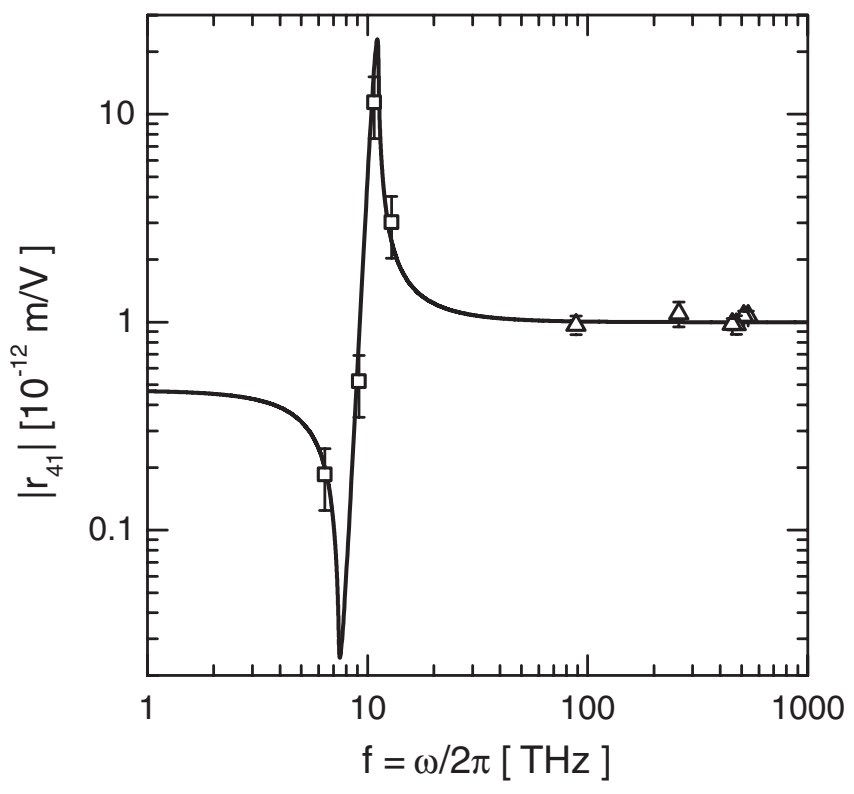

FIG. 8. Absolute magnitude of the EO coefficient $r_{41}(\omega)$ of GaP. The square symbols $\square$ are experimental data taken from Ref. [17], the triangles $\triangle$ from Ref. [33]. The curve is a fit of the experimental data using Eq. (24) with the parameters shown in the text.

Fig. 9 has been computed with the following parameters: $d_{E}=4.25 \times 10^{-12} \mathrm{~m} / \mathrm{V}$ and $C=-0.07$. The value of $C$ has been taken from Ref. [22]. In our simulations of the EO process in $\mathrm{ZnTe}$, we have used a constant EO coefficient of

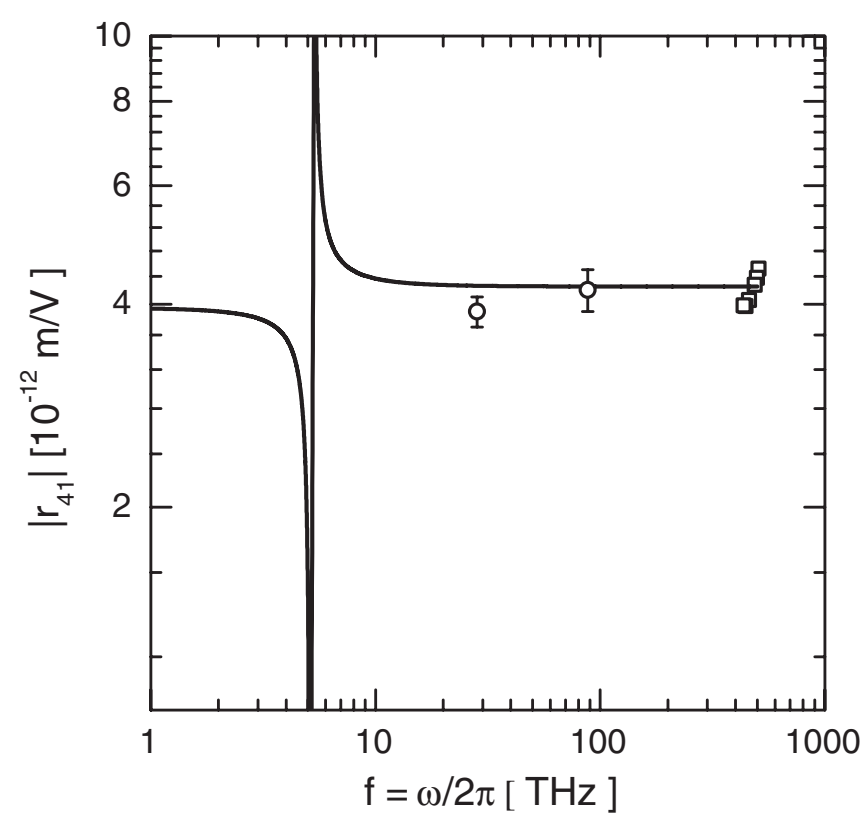

FIG. 9. The EO coefficient $\left|r_{41}\right|$ of ZnTe. The square symbols $\square$ are experimental data taken from Ref. [20] $(\rho \approx$ $\left.4 \times 10^{6} \Omega \mathrm{m}\right)$, the circles $\bigcirc$ from Ref. [19] ( $\left.\rho \approx 10^{7} \Omega \mathrm{m}\right)$. The curve is obtained using Eq. (24) with the parameters shown in the text. 
$r_{41}(\omega)=4.0 \times 10^{-12} \mathrm{~m} / \mathrm{V}$. We have verified that no significant change in the simulated EO signal is observed if Eq. (24) is used instead.

\section{SIMULATION OF THE ELECTRO-OPTIC PROCESS}

The equivalent $\mathrm{THz}$ pulse, corresponding to the transient electric field of the passing electron bunch, and the optical laser pulse are depicted in Fig. 10. The equivalent THz pulse is a truly unipolar pulse without superimposed highfrequency oscillations while the Ti:Sa laser pulse is a short wave train with a central wavelength of $\lambda_{0}=800 \mathrm{~nm}$ and a bandwidth of some $50 \mathrm{~nm}$. In the following sections only the envelope of the laser pulse will be shown.

\section{A. Phase and group velocities}

In the optical regime the refractive index $n$ of GaP and ZnTe decreases with increasing wavelength. The short Ti:Sa laser pulses propagate with the optical group velocity

$$
v_{g}=\frac{c}{n}\left(1+\frac{\lambda}{n} \frac{\mathrm{d} n}{\mathrm{~d} \lambda}\right)
$$

One finds $v_{g} / c=0.28$ for $\mathrm{GaP}$ and $v_{g} / c=0.30$ for $\mathrm{ZnTe}$.

The phase velocities of $\mathrm{GaP}$ and $\mathrm{ZnTe}$ in the $\mathrm{THz}$ range are shown in Fig. 11 as functions of frequency. For comparison also the optical group velocity at $\lambda=800 \mathrm{~nm}$ is plotted. While at low frequency a harmonic $\mathrm{THz}$ wave propagates with a somewhat higher speed than the laser pulse, the phase velocity drops steeply with increasing $f$, and there is a growing velocity mismatch when one approaches the TO resonance of $5.3 \mathrm{THz}$ in $\mathrm{ZnTe}$ and $11 \mathrm{THz}$ in $\mathrm{GaP}$.

\section{B. Pulse propagation}

To describe the EO effect, we use first a physically intuitive approach in which both the equivalent $\mathrm{THz}$ pulse and the optical laser pulse are propagated as wave packets through the EO crystal. The normal to the (110) plane is chosen as the $z$ direction. In this section the spreading of the laser pulse is neglected and a delta-function-like pulse with constant shape is moved through the EO crystal with the optical group velocity. The corresponding procedure is not adequate for the $\mathrm{THz}$ pulse since severe shape distortions happen when these pulses are very short. Therefore the $\mathrm{THz}$ pulse is decomposed into its Fourier components and these are propagated through the crystal with their respective phase velocity. The attenuation is taken into account. At selected positions $z_{j}$ inside the crystal the $\mathrm{THz}$ pulse shapes are reconstructed by inverse Fourier transformation.

For highly relativistic electrons with a Lorentz factor $\gamma>1000$, the electric field of the bunch is concentrated in a flat disk perpendicular to the direction of motion with an
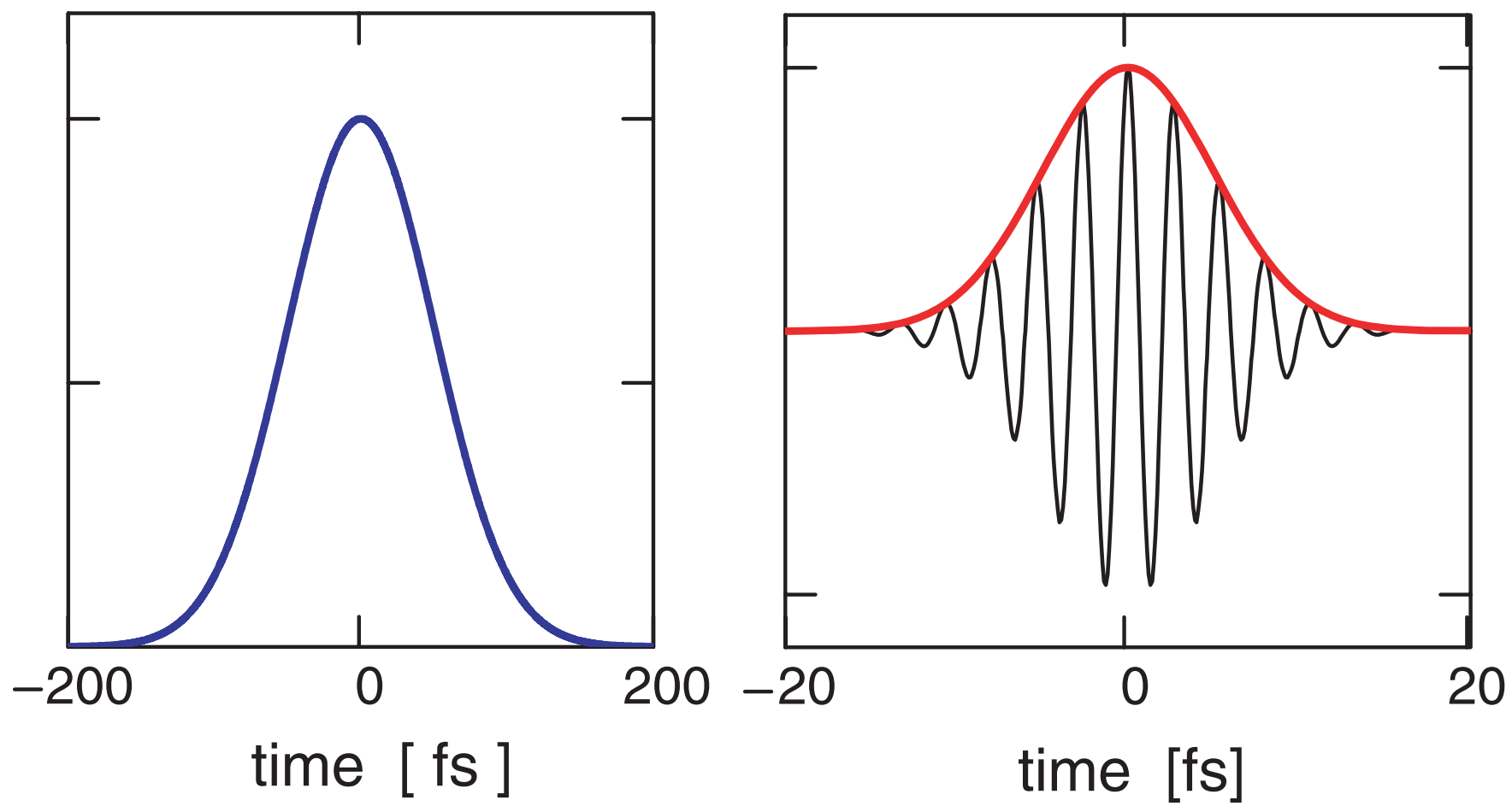

FIG. 10. (Color) Left: Sketch of the unipolar THz pulse corresponding to the transient electric field of a relativistic Gaussian electron bunch with $\sigma=50$ fs. Right: Envelope (solid red curve) and high-frequency oscillation (black) of a typical Ti:Sa laser pulse used for electro-optic sampling. 

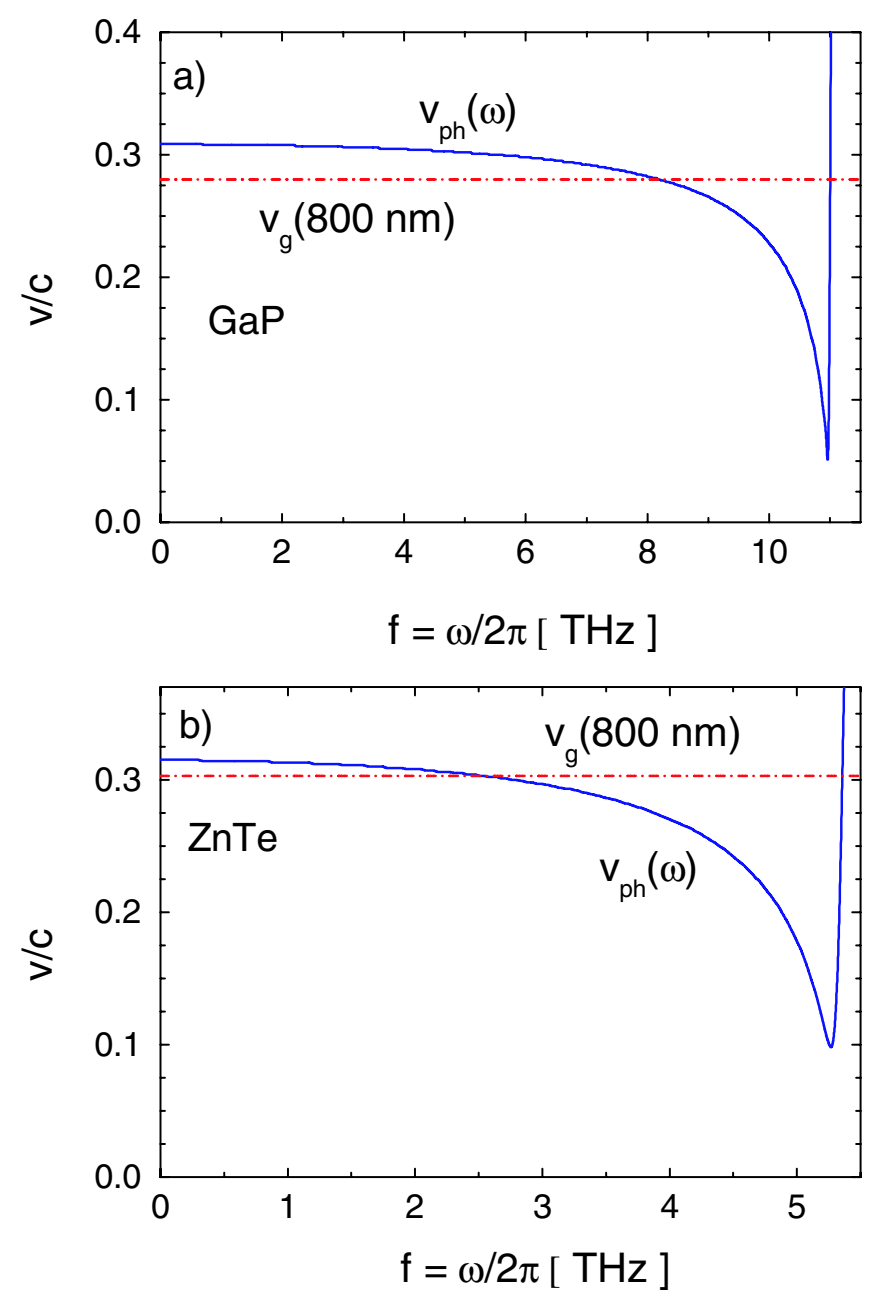

FIG. 11. (Color) (a) Phase velocity of GaP in the $\mathrm{THz}$ range. The optical group velocity at $\lambda=800 \mathrm{~nm}$ is shown as a dashdotted line. The velocities have been divided by $c$. (b) THz phase velocity and optical group velocity of ZnTe.

opening angle of $1 / \gamma$. The field has mainly a radial component. The EO crystal is mounted at a small distance $r$ from the beam. When the condition

$$
r / \gamma \ll L
$$

is fulfilled ( $L$ being the bunch length), the electric field pulse as seen by the EO crystal provides a faithful image of the electron bunch time profile.

The best insight into the capabilities and limitations of electro-optic bunch shape reconstruction is provided by studying Gaussian $\mathrm{THz}$ pulses. The electric field pulse at the EO crystal is thus assumed to have the time dependence

$$
E(t)=E_{0} \exp \left(-\frac{t^{2}}{2 \sigma^{2}}\right),
$$

where $\sigma$ is the rms duration of the THz pulse. If condition (26) is satisfied, the electron bunch profile is Gaussian as well with the same $\sigma$, and the amplitude $E_{0}$ can be calcu- lated from the bunch charge $Q$ by

$$
E_{0}=\frac{Q}{(2 \pi)^{3 / 2} \epsilon_{0} r c \sigma} .
$$

We call $F_{E}(\omega)$ the Fourier transform of the electric field pulse

$$
F_{E}(\omega)=\int_{-\infty}^{\infty} e^{i \omega t} E(t) \mathrm{d} t
$$

which in this special case can be computed analytically or, for more complicated charge distributions, by a fast Fourier transform (FFT) algorithm. At the interface between the accelerator vacuum and the EO crystal, some fraction of the incident wave is reflected, the remaining part is transmitted into the dielectric crystal. The amplitude transmission coefficient depends on frequency and is given by the expression

$$
A_{\mathrm{tr}}(\omega)=\frac{2}{n(\omega)+i \kappa(\omega)+1} .
$$

The Fourier component of the transmitted electric field pulse is

$$
F_{\mathrm{tr}}(\omega)=F_{E}(\omega) A_{\mathrm{tr}}(\omega) .
$$

To illustrate the pulse propagation method we subdivide the crystal into $N$ thin slices of thickness $\Delta z=d / N$. The Fourier components in the center of the slice $j$ at the

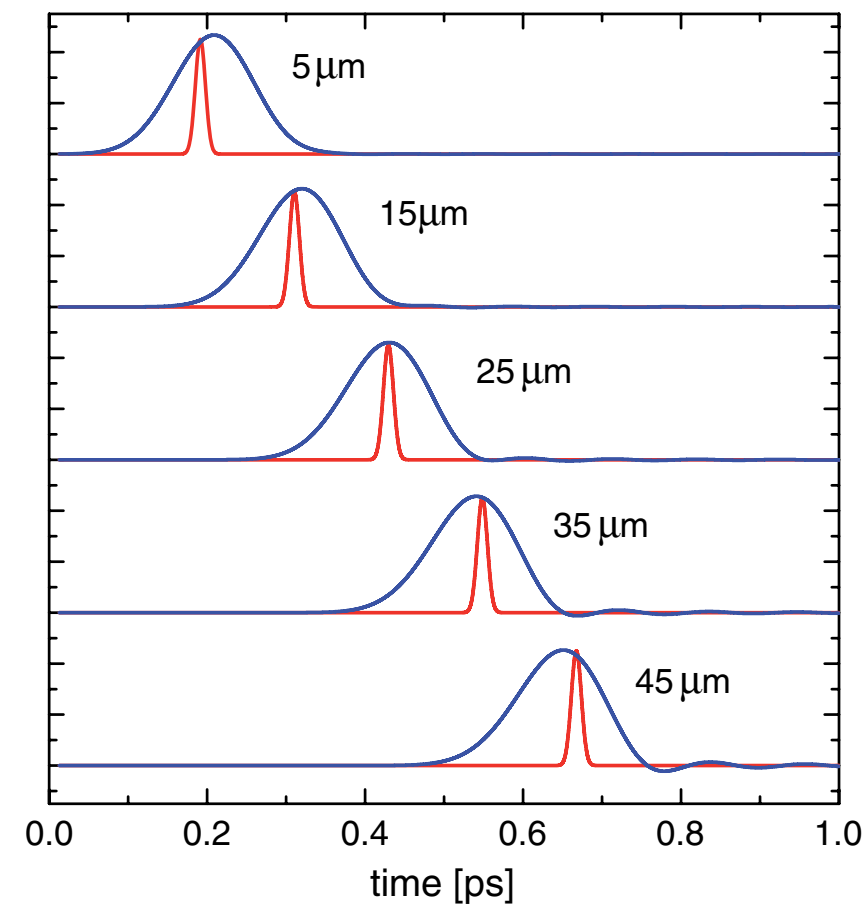

FIG. 12. (Color) Computed propagation of a Gaussian $\mathrm{THz}$ pulse with an initial rms width of $\sigma=50 \mathrm{fs}$ in a $50 \mu \mathrm{m}$ thick $\mathrm{GaP}$ crystal. The blue curves show the time profiles at selected positions in the crystal $\left(z_{j}=5,15,25,35,45 \mu \mathrm{m}\right)$. The positions of the laser pulses are shown as well (narrow red curves). 
position $z_{j}=(j-0.5) \Delta z$ are given by

$$
F_{j}^{\text {slice }}(\omega)=F_{\mathrm{tr}}(\omega) e^{i k z_{j}} e^{-\alpha z_{j}}
$$

where $k \equiv k(\omega)=\omega n(\omega) / c$ is the wave number in the refractive medium and $\alpha \equiv \alpha(\omega)=\omega \kappa(\omega) / c$ the frequency-dependent attenuation coefficient. The phase propagation in Eq. (32) is determined by the real part of the refractive index, $n(\omega)$, and the attenuation is determined by the imaginary part, $\kappa(\omega)$. The time profile of the pulse at slice $j$ is obtained by applying the inverse Fourier transformation to Eq. (32) and taking the real part:

$$
\begin{aligned}
E_{j}(t) & =\operatorname{Re}\left\{\frac{1}{2 \pi} \int_{-\infty}^{\infty} e^{-i \omega t} F_{j}^{\text {slice }}(\omega) \mathrm{d} \omega\right\} \\
& =\operatorname{Re}\left\{\frac{1}{\pi} \int_{0}^{\infty} e^{-i \omega t} F_{j}^{\text {slice }}(\omega) \mathrm{d} \omega\right\}
\end{aligned}
$$

As a first example for the pulse propagation method we consider a fairly long THz pulse with $\sigma=50$ fs which is passing through a very thin GaP crystal of thickness $d=$ $50 \mu \mathrm{m}$. The crystal is subdivided into 25 slices. The time profile of the pulse at five selected positions in the crystal $\left(z_{j}=5,15,25,35,45 \mu \mathrm{m}\right)$ is shown in Fig. 12. Owing to its rather large width the $\mathrm{THz}$ pulse retains its shape while propagating in the EO crystal. The laser pulse needs a slightly longer time to travel through the crystal but the temporal overlap of the two pulses is fair in all slices.

Next we take a 10 times thicker GaP crystal. Figure 13 shows that in this case the velocity mismatch is severe. The relative timing has been chosen such that laser pulse and $\mathrm{THz}$ pulse are coincident at a depth of $50 \mu \mathrm{m}$. Then the two pulses have a fairly good overlap in the first $100 \mu \mathrm{m}$ thick layer of the GaP crystal; however, the laser pulse is far too late in the remaining $400 \mu \mathrm{m}$ thick section of the crystal. This means that the largest part of the EO crystal does not contribute to the EO signal; on the contrary, it has a rather negative impact on the phase retardation signal $\Gamma$ accumulated in the first layer.

\section{Response function}

The mismatch between the $\mathrm{THz}$ phase velocity and the optical group velocity of the laser pulse that is visible in Fig. 13 degrades the quality of the EO pulse shape reconstruction and limits the tolerable EO crystal thickness. To obtain a quantitative measure of this velocity mismatch we define a geometric response function by

$$
\begin{aligned}
G(\omega) & =\frac{1}{d} \int_{0}^{d}\left[\int e^{i[k z-\omega t]} e^{-\alpha z} \delta\left(z / v_{g}-t\right) \mathrm{d} t\right] \mathrm{d} z \\
& =\frac{1}{d} \int_{0}^{d} \exp \left(\frac{i \omega z}{v_{\mathrm{ph}}(\omega)}-\frac{i \omega z}{v_{g}}\right) e^{-\alpha z} \mathrm{~d} z .
\end{aligned}
$$

$G(\omega)$ depends on the thickness $d$ of the EO crystal. The geometric response function is close to unity if the velocities are well matched. The computed geometric response

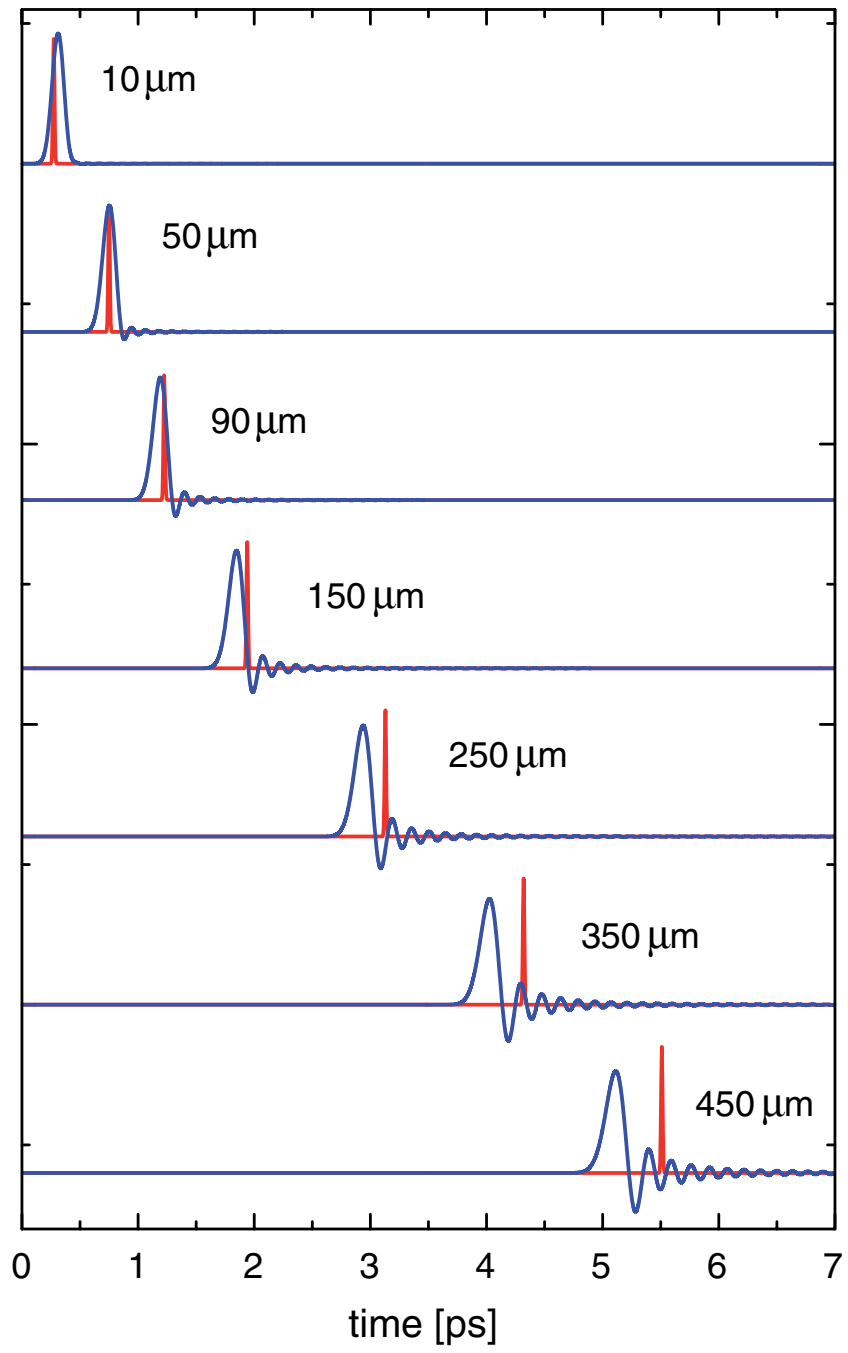

FIG. 13. (Color) Computed propagation of a Gaussian $\mathrm{THz}$ pulse with an initial rms width of $\sigma=50 \mathrm{fs}$ in a $500 \mu \mathrm{m}$ thick $\mathrm{GaP}$ crystal. Plotted are the time profiles at selected positions in the crystal (blue curves). The leading edge is at the left side. The positions of the laser pulses are shown by the narrow red curves.

function of GaP is shown in Fig. 14, the response function of $\mathrm{ZnTe}$ is depicted in Fig. 15.

We note that the response function of a $200 \mu \mathrm{m}$ thick $\mathrm{GaP}$ crystal becomes very small at frequencies between 5 and $6 \mathrm{THz}$. For a $d \approx 230 \mu \mathrm{m}$ crystal, the response function would even vanish near $5.5 \mathrm{THz}$. The explanation is that the phase factor appearing in the integral (34) carries out a full $360^{\circ}$ rotation in the complex plane when $z$ grows from zero to $230 \mu \mathrm{m}$, and hence the integral over this phase factor tends to zero. The consequence is that a $d=$ $230 \mu \mathrm{m} \mathrm{GaP}$ crystal would yield a tiny EO signal when a sinusoidal $5.5 \mathrm{THz}$ wave is scanned by the laser pulse. In our pulse propagation picture we can understand this as follows. If one adjusts the timing of the laser pulse such that it coincides with the center of a positive half wave at a depth of $z=d / 4$ in the crystal, then the phase slippage is 


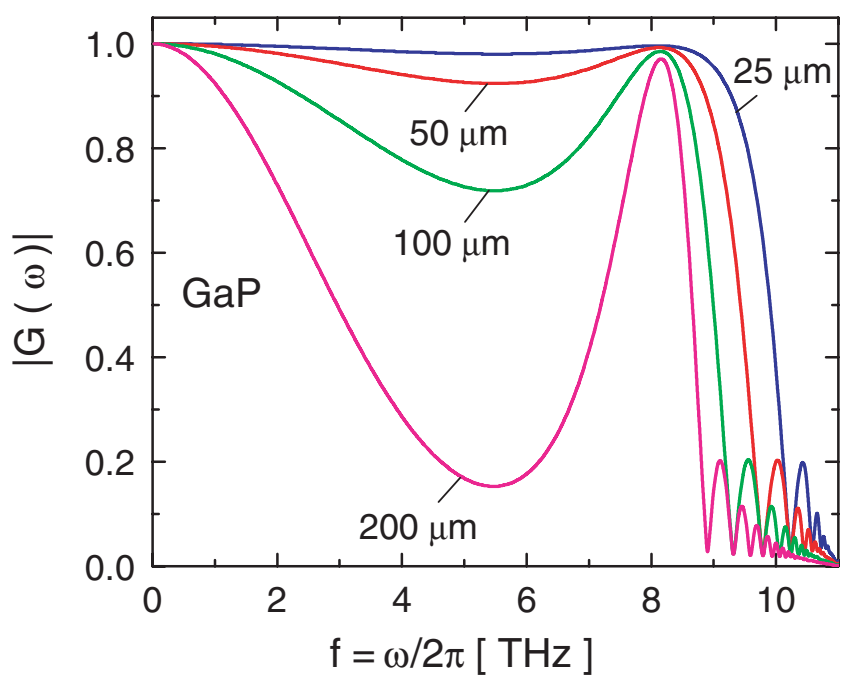

FIG. 14. (Color) Absolute magnitude of the geometric response function of $\mathrm{GaP}$ for a crystal thickness of 25, 50, 100, and $200 \mu \mathrm{m}$.

such that the laser pulse will coincide with the center of a negative half wave at $z=3 d / 4$. In other words: the positive phase retardation $\Gamma$ accumulated in the first half of the $\mathrm{GaP}$ crystal will be largely canceled by the negative phase retardation $\Gamma$ accumulated in the second half. Similar cancellation effects happen in ZnTe and are responsible for the dips in the response function. A dip around $1.5 \mathrm{THz}$ was previously reported in Fig. 2 of Ref. [16] for a $2 \mathrm{~mm}$ thick ZnTe crystal.

From these observations it is obvious that high $\mathrm{THz}$ frequencies can only be reached in sufficiently thin crystals. In ZnTe, for example, the TO resonance at $5.3 \mathrm{THz}$ and the velocity mismatch set an upper limit of about

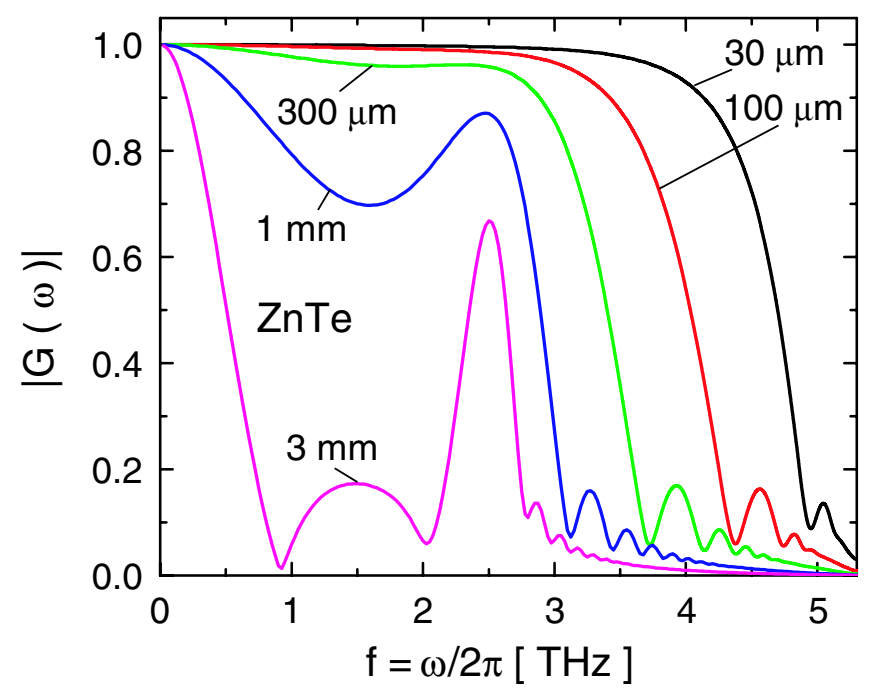

FIG. 15. (Color) Absolute magnitude of the geometric response function of ZnTe for a crystal thickness of $30 \mu \mathrm{m}, 100 \mu \mathrm{m}$, $300 \mu \mathrm{m}, 1 \mathrm{~mm}$, and $3 \mathrm{~mm}$.
3.7 $\mathrm{THz}$ to the accessible frequency range for a crystal thickness of up to $300 \mu \mathrm{m}$. Gallium phosphide permits one to double the frequency range, but it is apparent from Fig. 14 that a fairly thin crystal $(d \leq 100 \mu \mathrm{m})$ should be used to exploit this capability. At too large a thickness, for example $d=200 \mu \mathrm{m}$, only about $4 \mathrm{THz}$ are reached in $\mathrm{GaP}$ which is then clearly inferior to $\mathrm{ZnTe}$ with its much larger EO coefficient $r_{41}$.

Note that in the literature (e.g. [23]) the amplitude transmission coefficient is often included in the definition of $G$. Since in case of GaP the electro-optic coefficient $r_{41}$ exhibits a strong frequency dependence we find it convenient to define an electro-optic response function by including the EO coefficient as well:

$$
G_{\mathrm{EO}}(\omega)=G(\omega) \cdot A_{\mathrm{tr}}(\omega) \cdot r_{41}(\omega)
$$

\section{Computation of the phase retardation}

In the section on pulse propagation, we have computed the shape of the THz pulse at various positions inside the EO crystal, and its relative timing with respect to the laser pulse. Equation (17) can be applied to calculate the retardation parameter $\Delta \Gamma_{j}$ for each slice. In the computation of $\Delta \Gamma_{j}$ the frequency dependence of the EO coefficient $r_{41}$ must be taken into account. This is done by multiplying the Fourier component of the propagated field pulse [Eq. (32)] with $r_{41}(\omega)$, and by applying the inverse FFT (IFFT). The resulting "effective" THz pulse [24] in slice $j$ is therefore given by

$$
E_{j}^{\text {eff }}(t)=\operatorname{Re}\left\{\frac{1}{\pi} \int_{0}^{\infty} e^{-i \omega t} F_{j}^{\text {slice }}(\omega) r_{41}(\omega) \mathrm{d} \omega\right\} .
$$

For small pulse duration the effective $\mathrm{THz}$ pulse acquires large oscillations following the main peak, owing to the fact that $\mathrm{EO}$ coefficient $r_{41}(\omega)$ of $\mathrm{GaP}$ approaches zero at $f=\omega /(2 \pi) \approx 8 \mathrm{THz}$ (see Fig. 18 for an illustration). One can see immediately from Fig. 12 that the phase retardation $\Delta \Gamma_{j}$ generated in the slice $\left[z_{i}-\Delta z / 2, z_{j}+\Delta z / 2\right]$ is proportional to the electric field amplitude $E^{\text {eff }}\left(z_{j}, t_{j}^{\text {las }}\right)$ at the arrival time of the laser pulse in that slice:

$$
\Delta \Gamma_{j}=\frac{2 \pi n_{0}^{3}}{\lambda_{0}} E^{\mathrm{eff}}\left(z_{j}, t_{j}^{\mathrm{las}}\right) \Delta z
$$

This arrival time is

$$
t_{j}^{\mathrm{las}}=\frac{z_{j}}{v_{g}}+\tau,
$$

where we have allowed for a variable time delay $\tau$ between $\mathrm{THz}$ and laser pulse. The delay will be varied in small steps in electro-optic sampling (EOS) experiments to map out the time profile of the $\mathrm{THz}$ pulse from measurements on many consecutive pulses. The total phase retardation accumulated in the EO crystal is the sum over the contributions from each slice: 


$$
\Gamma(\tau)=\frac{2 \pi n_{0}^{3}}{\lambda_{0}} \sum_{j=1}^{N} E^{\mathrm{eff}}\left(z_{j}, \frac{z_{j}}{v_{g}}+\tau\right) \Delta z
$$

If the number of slices tends to infinity the sum (37) goes over into an integral. Using Eqs. (34) and (36) we find that the total phase retardation can be computed from the electro-optic response function [see Eq. (35)] by an inverse Fourier transformation:

$$
\Gamma(\tau)=\frac{2 n_{0}^{3} d}{\lambda_{0}} \operatorname{Re}\left\{\int_{0}^{\infty} e^{-i \omega \tau} F_{E}(\omega) G_{\mathrm{EO}}(\omega) \mathrm{d} \omega\right\} .
$$

The equations (37) and (38) have been derived under the assumption that a very narrow, almost delta-function-like, Ti:Sa laser pulse moves through the EO crystal without shape distortion or broadening. In our numerical simulations the Fourier transforms and the inverse transforms are made using FFT and IFFT algorithms.

\section{E. Multiple reflections in the EO crystal}

The $\mathrm{THz}$ pulse can undergo a reflection at the rear surface of the crystal, move back, and after a second reflection at the front surface, move again through the crystal in the forward direction. This is sometimes referred to as the Fabry-Perot effect. The double-reflected pulse will be scanned if the laser pulse is delayed by the travel time $2 d / v_{g}^{\mathrm{THz}}$ (here $v_{g}^{\mathrm{THz}}$ is the group velocity in the frequency range covered by the $\mathrm{THz}$ pulse). The Fourier transform of the twice-reflected $\mathrm{THz}$ pulse, just behind the front surface of the $\mathrm{EO}$ crystal, is given by

$$
F_{2}(\omega)=F_{\text {tr }}(\omega) A_{\text {ref }}^{2}(\omega) e^{i 2 k d} e^{-2 \alpha d},
$$

where $\quad A_{\text {ref }}(\omega)=[1-n(\omega)-i \kappa(\omega)] /[1+n(\omega)+$ $i \kappa(\omega)]$ is the amplitude reflection coefficient. These Fourier components are propagated to the slices of the crystal by the same method that was applied for the direct $\mathrm{THz}$ pulse. The phase retardation signal in Fig. 16 shows the direct signal, and after a delay time of $\delta t=2 d / v_{g}^{\mathrm{THz}}$, the signal from the double-reflected $\mathrm{THz}$ pulse. This second signal is much weaker, about $20 \%$ of the first peak, mainly due to the reflection coefficient which enters quadratically. The next reflection will produce a pulse at twice the delay but with a signal amplitude of only a few percent of the main peak. This and even higher-order reflections will be easily lost in the noise, so we refrain from summing up the Fabry-Perot effect in a geometric series.

Also the laser pulse will undergo multiple reflections in the EO crystal. If the double-reflected laser pulse coincides with the double-reflected $\mathrm{THz}$ pulse, one gets a contribution to the balanced detector signal at the position of the first main peak. This contribution is small since not only the $\mathrm{THz}$ pulse but also the laser pulse is attenuated by the double reflection. The effect can be avoided altogether if one uses a wedge-shaped EO crystal. The EO crystal then acts like a prism and deflects the direct laser beam by an

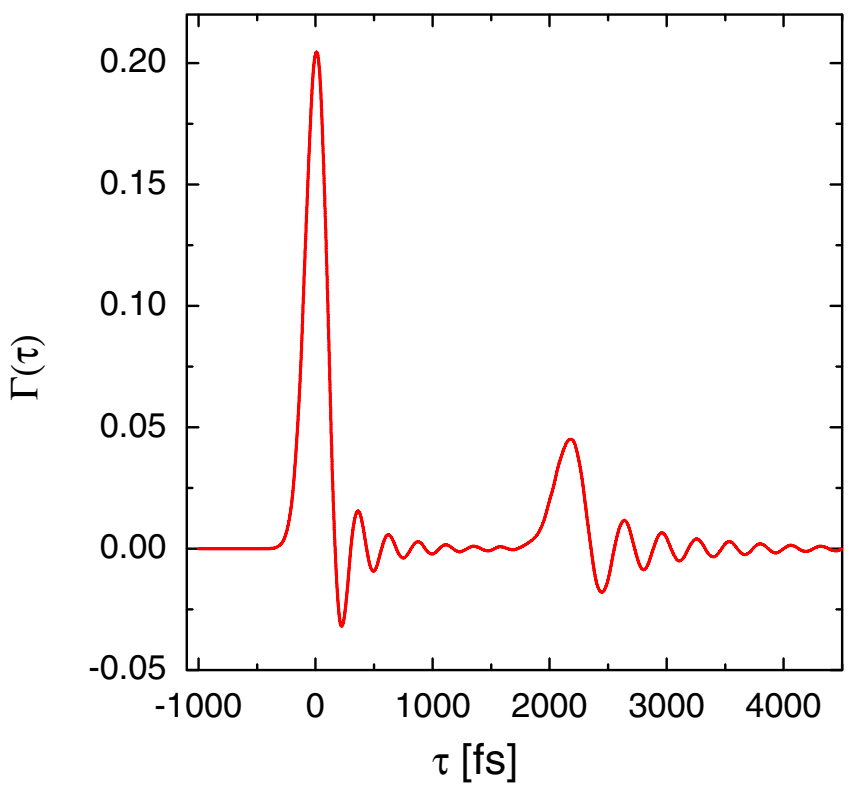

FIG. 16. (Color) The balanced detector signal of a Gaussian THz pulse with $\sigma=70 \mathrm{fs}$ in a $100 \mu \mathrm{m}$ thick ZnTe crystal. The bunch charge is $Q=0.1 \mathrm{nC}$ and the distance between the electron beam and the crystal is $r=5 \mathrm{~mm}$. Both the direct and the double-reflected pulse are shown.

angle of $\left(n_{0}-1\right) \phi$, where $\phi$ is the wedge angle and $n_{0}$ the refractive index for laser light. The double-reflected laser beam will leave the EO crystal at a deflection angle of $\left(3 n_{0}-1\right) \phi$ and can therefore be easily separated from the direct laser beam. Note, however, that the wedge angle does not eliminate the multiple reflections of the $\mathrm{THz}$ pulse. The reason is that the electric field carried by the electron bunch is not well collimated like the Ti:Sa laser beam, but extends on the whole EO crystal.

\section{F. Consideration of laser-pulse broadening}

The next refinement is to decompose the broadband laser pulse into its Fourier components and propagate these through the EO crystal. A complete frequency-domain description of electro-optic detection is presented in Ref. [25]. Under the justified assumptions that the time duration of the laser pulse is significantly shorter than the temporal features of the effective $\mathrm{THz}$ pulse and the laser bandwidth $\Delta \omega$ is much less than the carrier frequency $\omega_{0}$, it can be shown [25] that a simplified treatment is possible in which the laser pulse moves through the EO crystal with the optical group velocity $v_{g}\left(\omega_{0}\right)$. The pulse width grows with increasing depth in the EO crystal [26]. The rms width in slice $j$ at the depth $z=z_{j}$ is

$$
\sigma_{j}=\sigma_{0} \sqrt{1+\left(\frac{z_{j}}{L_{\text {char }}}\right)^{2}}
$$

with a characteristic length 


$$
L_{\mathrm{char}}=-2 \sigma_{0}^{2} v_{g}^{2}\left[\frac{\mathrm{d} v_{g}}{\mathrm{~d} \omega}\right]^{-1}
$$

One obtains $L_{\text {char }}=30 \mu \mathrm{m}$ in ZnTe for Ti:Sa laser pulses with a FWHM duration of $15 \mathrm{fs}\left(\sigma_{0}=6.4 \mathrm{fs}\right)$. The overlap of the $\mathrm{THz}$ and laser pulses in each slice is computed by a convolution integral. The phase retardation as a function of the variable delay time $\tau$ between $\mathrm{THz}$ and laser pulse is then

$$
\begin{aligned}
\Gamma(\tau)= & \frac{2 \pi n_{0}^{3} \Delta z}{\lambda_{0}} \frac{1}{\sqrt{2 \pi}} \sum_{j=1}^{N} \int E_{j}^{\mathrm{eff}}\left(z_{j}, \frac{z_{j}}{v_{g}}+t\right) \frac{1}{\sigma_{j}} \\
& \times \exp \left(-\frac{(t-\tau)^{2}}{2 \sigma_{j}^{2}}\right) \mathrm{d} t .
\end{aligned}
$$

In Fig. 17 we show the expected EO signal as a function of the relative delay $\tau$ between the $\mathrm{THz}$ and the laser pulse. The computations have been made for a $500 \mu \mathrm{m}$ thick ZnTe crystal and a THz pulse with $\sigma=70 \mathrm{fs}$. The influence of laser-pulse broadening in the rather thick EO crystal is visible but not very significant. For thinner crystals it can be neglected so it is legitimate to use Eq. (38) for computing the phase retardation parameter $\Gamma$.

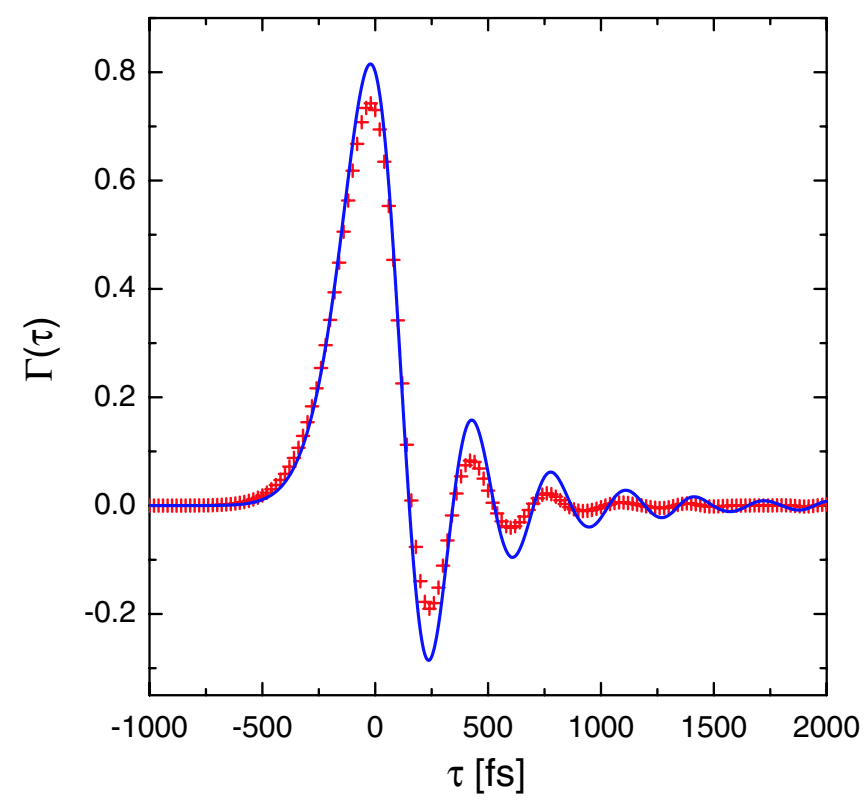

FIG. 17. (Color) Effect of laser-pulse broadening in a $500 \mu \mathrm{m}$ thick ZnTe crystal which is placed at a distance $r=5 \mathrm{~mm}$ from an electron beam with bunch charge $Q=0.1 \mathrm{nC}$ and $\mathrm{rms}$ bunch duration $\sigma_{t}=70$ fs. Plotted is the phase retardation $\Gamma(\tau)$ as a function of the relative delay $\tau$ between the $\mathrm{THz}$ pulse and the laser pulse. Continuous blue curve: delta-function-like laser pulse, Eq. (38). Crosses: laser-pulse broadening taken into consideration according to Eq. (40). The initial laser-pulse length is $\sigma_{0}=6.4 \mathrm{fs}$.

\section{QUALITY OF ELECTRO-OPTIC BUNCH SHAPE RECONSTRUCTION}

\section{A. Usable frequency range}

The electro-optic detection of $\mathrm{THz}$ pulses depends strongly on the frequency spectrum of the pulses. The important issue is whether the spectral components of the pulses are predominantly below or above the TO lattice resonance of the EO crystal. The first case applies to most $\mathrm{THz}$ spectroscopy experiments and to the EO detection of short electron bunches. The transient electric field pulse of a relativistic electron bunch constitutes a truly unipolar $\mathrm{THz}$ pulse whose Fourier components range from zero up to many $\mathrm{THz}$, depending on the bunch length. The dc and the low-frequency components are always present and are usually dominant. The situation is quite different in some very high-frequency $\mathrm{THz}$ experiments where the $\mathrm{THz}$ pulses are generated from nonresonant optical rectification of ultrashort laser pulses. Such $\mathrm{THz}$ pulses have frequency components mainly above the TO resonance, see Fig. 5 in Ref. [5] and Fig. 1 in Ref. [27] as examples.

In our simulations we have modeled the EO crystal by a resonating system that is driven by a periodic force. Well below or well above the eigenfrequency of the resonating system the frequency-response curve will be flat or only slowly varying, while close to resonance it exhibits strong variations. For very high-frequency $\mathrm{THz}$ applications such as reported in [27,28], the frequency range above the TO resonance is relevant and time resolutions in the order of $10 \mathrm{fs}$ can be achieved.

The situation is entirely different in the electro-optic experiments at electron accelerators. Here the frequency range below the lattice resonance is relevant with the consequence that extremely short electron bunches with an rms duration in the order of $10 \mathrm{fs}$ cannot be faithfully reconstructed. The equivalent $\mathrm{THz}$ pulses of such ultrashort bunches have large Fourier components in the resonance region, and these will be resonantly amplified and will overshadow the weaker Fourier components above resonance. An example is shown in Fig. 18 for a $\sigma=$ 10 fs pulse [29] propagating through a $50 \mu \mathrm{m} \mathrm{GaP}$ crystal. The propagated pulses bear little resemblance to the incident $\mathrm{THz}$ pulses. Strong oscillations are observed lagging behind the main signal, but there are even oscillations ahead of this signal due to the fact that the $\mathrm{THz}$ phase velocity exhibits a strong rise at resonance (see Fig. 11). Similar strong oscillations have been reported in Ref. [5] for $\mathrm{THz}$ pulses generated by $12 \mathrm{fs}$ Ti:Sa laser pulses incident on a GaAs crystal, and recorded in a $30 \mu \mathrm{m}$ $\mathrm{ZnTe}$ crystal. An unfolding of the EO frequency characteristic near resonance appears virtually impossible due to the imprecise knowledge of the complex refractive index $n(\omega)+i \kappa(\omega)$ and the even much less precise knowledge of $r_{41}(\omega)$. We are presently not aware of a simple method that would allow one to utilize the frequency components 

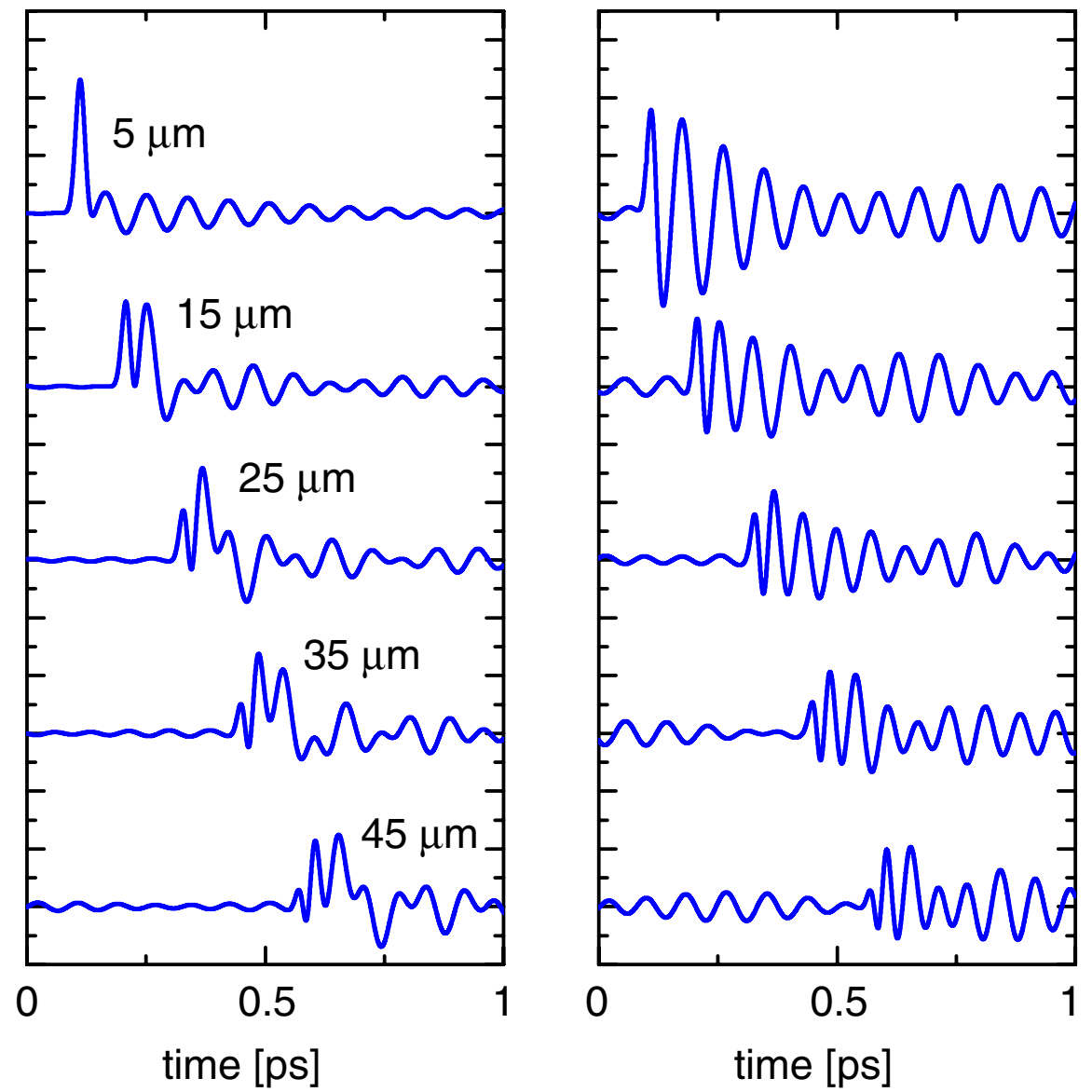

FIG. 18. (Color) Left: Propagation of a Gaussian THz pulse with an initial rms width of $\sigma=10$ fs in a $50 \mu \mathrm{m}$ thick GaP crystal. The numbers in the plot indicate the respective position $z_{j}$ in $\mu \mathrm{m}$. Right: The shape of the effective THz pulse according to Eq. (36), taking into account the frequency dependence of $r_{41}$.

above resonance in order to reach a 10 fs resolution in electron bunch diagnostics.

\section{B. Comparison of original bunch shape and computed EO signal}

In this section we consider again electron bunches with a Gaussian longitudinal charge distribution. The analysis procedure for a Gaussian $\mathrm{THz}$ pulse has been explained in the previous section. Here we summarize the simulation results for a $d=65 \mu \mathrm{m} \mathrm{GaP}$ crystal, such as used in Ref. [4], and $\mathrm{THz}$ pulses of various rms width. The bunch charge is chosen to be $Q=0.1 \mathrm{nC}$, and the distance between the electron beam and the spot on the EO crystal which is scanned by the laser is taken as $r=5 \mathrm{~mm}$.

The expected signal in a balanced detector setup is proportional to $\sin [\Gamma(\tau)] \approx \Gamma(\tau)$. The computed signal $\Gamma(\tau)$ as function of the delay time $\tau$ is compared in Fig. 19 with the original $\mathrm{THz}$ pulse shape. A long $\mathrm{THz}$ pulse with $\sigma \geq 70 \mathrm{fs}$ is almost perfectly reconstructed. No oscillations are observed in the tail of the detector signal since such a pulse has very small Fourier components near the $\mathrm{TO}$ resonance of $\mathrm{GaP}$ at $11 \mathrm{THz}$. At $\sigma=50 \mathrm{fs}$ the reconstructed signal is slightly broader. Significant pulse stretching associated with oscillations following the main peak is observed for $\sigma \leq 30$ fs. The pulse shape distortions become very severe for even shorter $\mathrm{THz}$ pulses with $\sigma=15 \mathrm{fs}$, respectively $10 \mathrm{fs}$, see the bottom part of Fig. 19. The phase retardation signal is much wider than the original bunch and its shape bears little resemblance to the initial Gaussian shape. These extremely short pulses are indeed inaccessible to a faithful reconstruction by the EO method using GaP.

If $\mathrm{ZnTe}$ is used instead of $\mathrm{GaP}$ a similar picture is obtained if one multiplies the $\mathrm{THz}$ pulse lengths with a factor of about two (the inverse ratio of the TO lattice frequencies). This is demonstrated in Fig. 20. A THz pulse with $\sigma=100 \mathrm{fs}$ is faithfully reconstructed using $\mathrm{ZnTe}$ while a 50 fs pulse appears significantly stretched, and the main peak is followed by oscillations.

In Fig. 21 we show the pulse stretching as a function of the initial pulse length. Since the EO signals of very short $\mathrm{THz}$ pulses do not possess a Gaussian shape but are severely distorted by oscillations, the characterization of the 

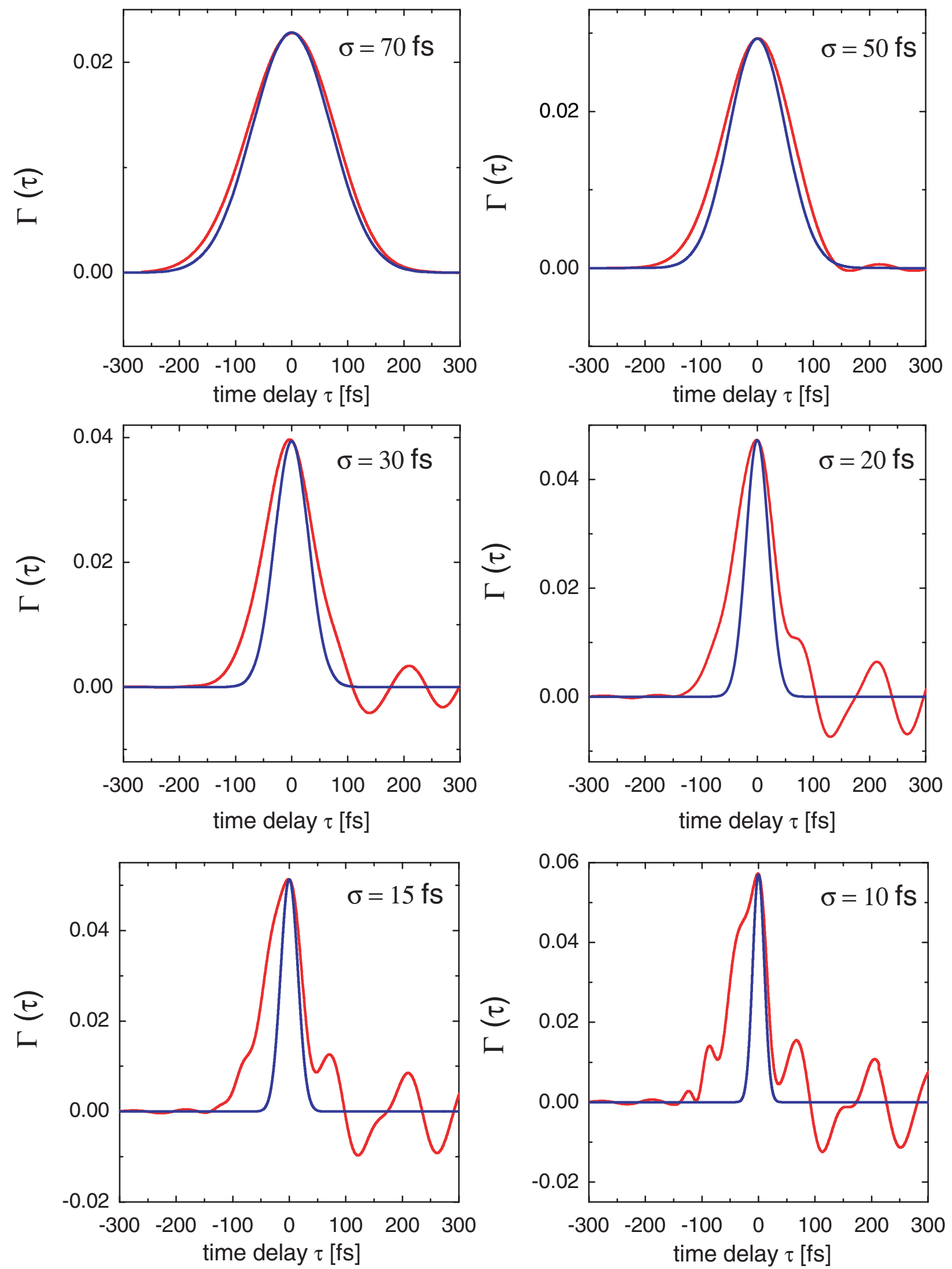

FIG. 19. (Color) EO pulse reconstruction using a $d=65 \mu \mathrm{m} \mathrm{GaP}$ crystal. The original Gaussian THz pulse is shown as a blue curve, the computed phase retardation signal $\Gamma(\tau)$ is drawn as a red curve. The initial $\mathrm{rms}$ is $\sigma=70,50,30,20,15,10 \mathrm{fs}$. 

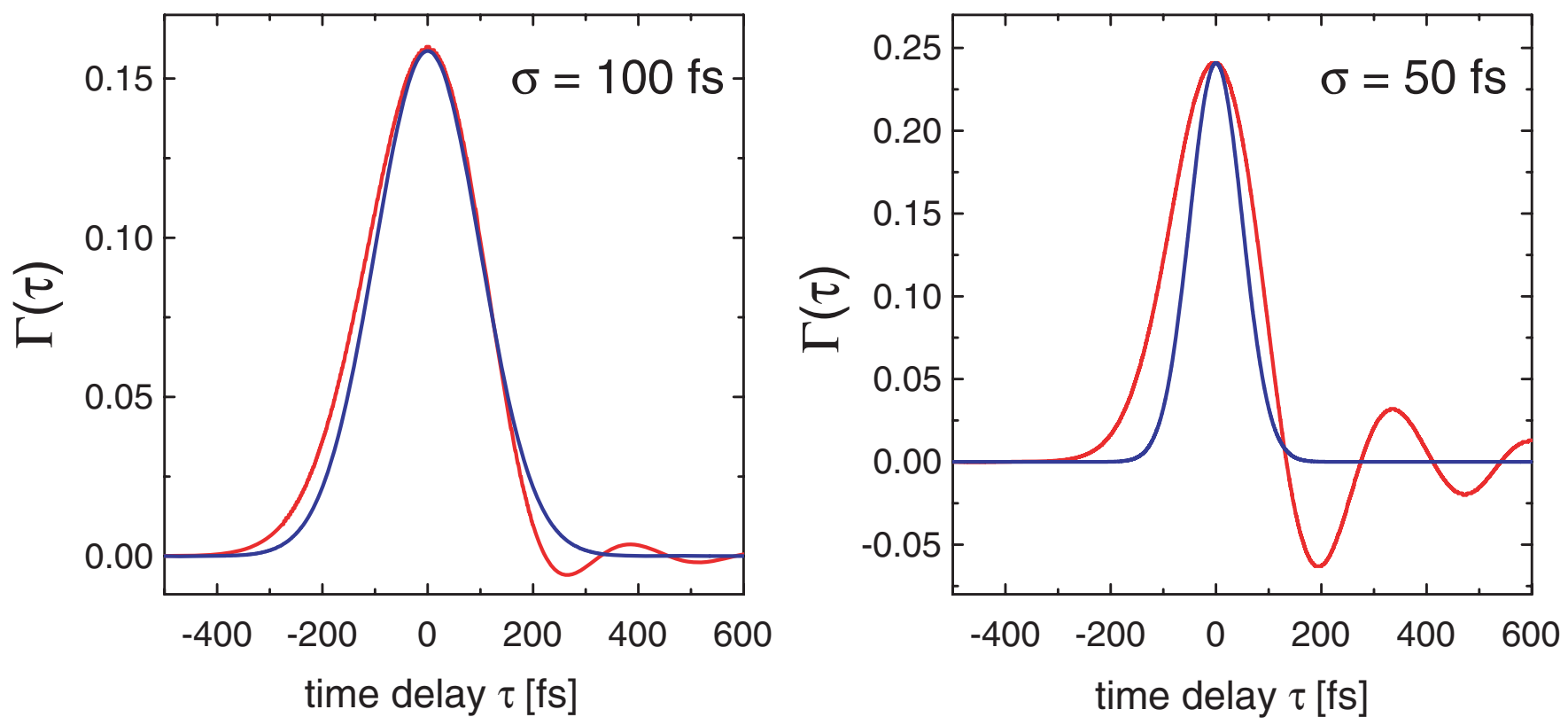

FIG. 20. (Color) EO pulse reconstruction using a $100 \mu \mathrm{m}$ ZnTe crystal and Gaussian THz pulses with $\sigma=100 \mathrm{fs}$, respectively 50 fs.

pulse shape by a single number is problematic. We have decided to compute the root-mean-square value $\sigma_{\mathrm{EO}}$ of the EO signal within a $500 \mathrm{fs}$ interval centered around the main peak. For an initial rms length of $\sigma_{\text {in }} \geq 100 \mathrm{fs}$, we find $\sigma_{\mathrm{EO}}=\sigma_{\text {in }}$ to a good approximation both for ZnTe and $\mathrm{GaP}$, hence both EO materials are well suited to reconstruct the original shape without noticeable distortion. In case of $\mathrm{ZnTe}, \sigma_{\mathrm{EO}}$ has a broad minimum of about 90 fs for initial pulse lengths below $90 \mathrm{fs}$. In case of $\mathrm{GaP}$ the minimum is lower, a little more than 50 fs, but below 30 fs the rms

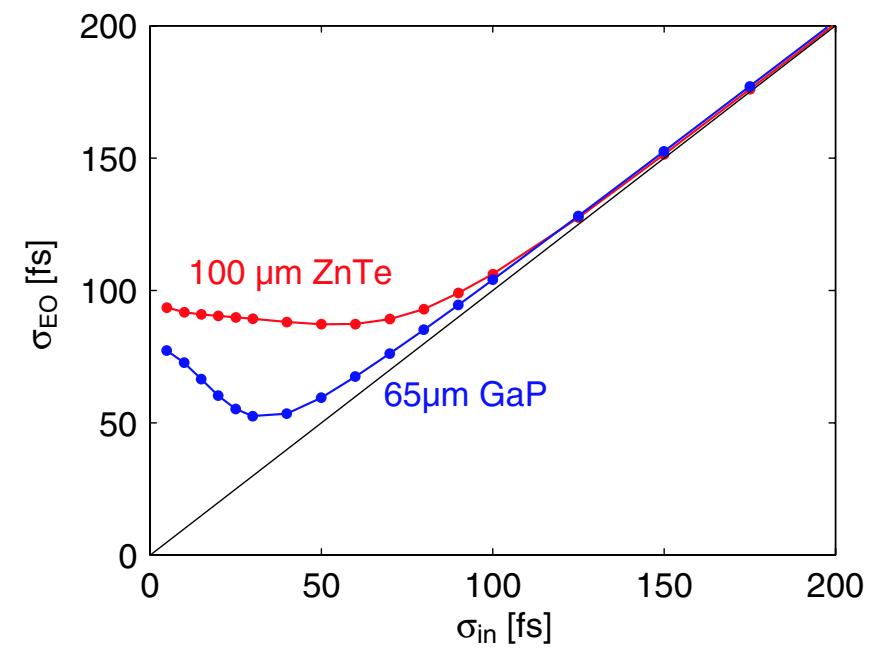

FIG. 21. (Color) Pulse stretching in a $65 \mu \mathrm{m}$ thick GaP crystal and a $100 \mu \mathrm{m}$ thick ZnTe crystal. The rms pulse width $\sigma_{\mathrm{EO}}$ of the simulated EO signal is plotted as a function of the initial rms width $\sigma_{\text {in }}$ of the equivalent $\mathrm{THz}$ pulse, representing the transient electric field of the electron bunch. length $\sigma_{\mathrm{EO}}$ grows due to the increasing amount of oscillations of the EO signal.

We conclude that the lowest rms bunch length which can be reconstructed with acceptable $(<20 \%)$ broadening amounts to about 50 fs in $\mathrm{GaP}$ and 90 fs in ZnTe. Note that we have assumed in our analysis ideal electro-optic sampling conditions in neglecting any time jitter between the electron bunches and laser pulses.

\section{Dependence of the EO signal on the crystal thickness}

Ideally, the amplitude of the EO signal should be directly proportional to the thickness $d$ of the EO crystal. For $\mathrm{ZnTe}$ this is fulfilled to a good approximation for a thickness of up to $400 \mu \mathrm{m}$, see Fig. 22. However, for $\mathrm{GaP}$ the more pronounced phase and group velocity mismatch leads to a slower than linear rise of the phase retardation signal with $d$ and eventually to a saturation. With increasing EO crystal thickness, the width of the EO signal grows considerably because the high-frequency Fourier components are suppressed (see Figs. 14 and 15). In order to preserve a good time resolution the GaP crystal thickness should therefore not exceed $d=100 \mu \mathrm{m}$. On the other hand, going to $d=50 \mu \mathrm{m}$ or less does not really improve the resolution but only leads to much smaller signals. The above figure suggests to use a GaP crystal thickness in the range $50 \mu \mathrm{m} \leq d \leq 100 \mu \mathrm{m}$ if one wants to detect electron bunches with a length in the sub- 100 fs regime. The small EO signal amplitude is then an unavoidable consequence. For ZnTe the conditions on crystal thickness are more relaxed. In case of short electron bunches ( $\sigma$ in the order of $100 \mathrm{fs}$ ), the optimum thickness is $100 \mu \mathrm{m} \leq$ $d \leq 300 \mu \mathrm{m}$ while for picosecond long electron bunches a 

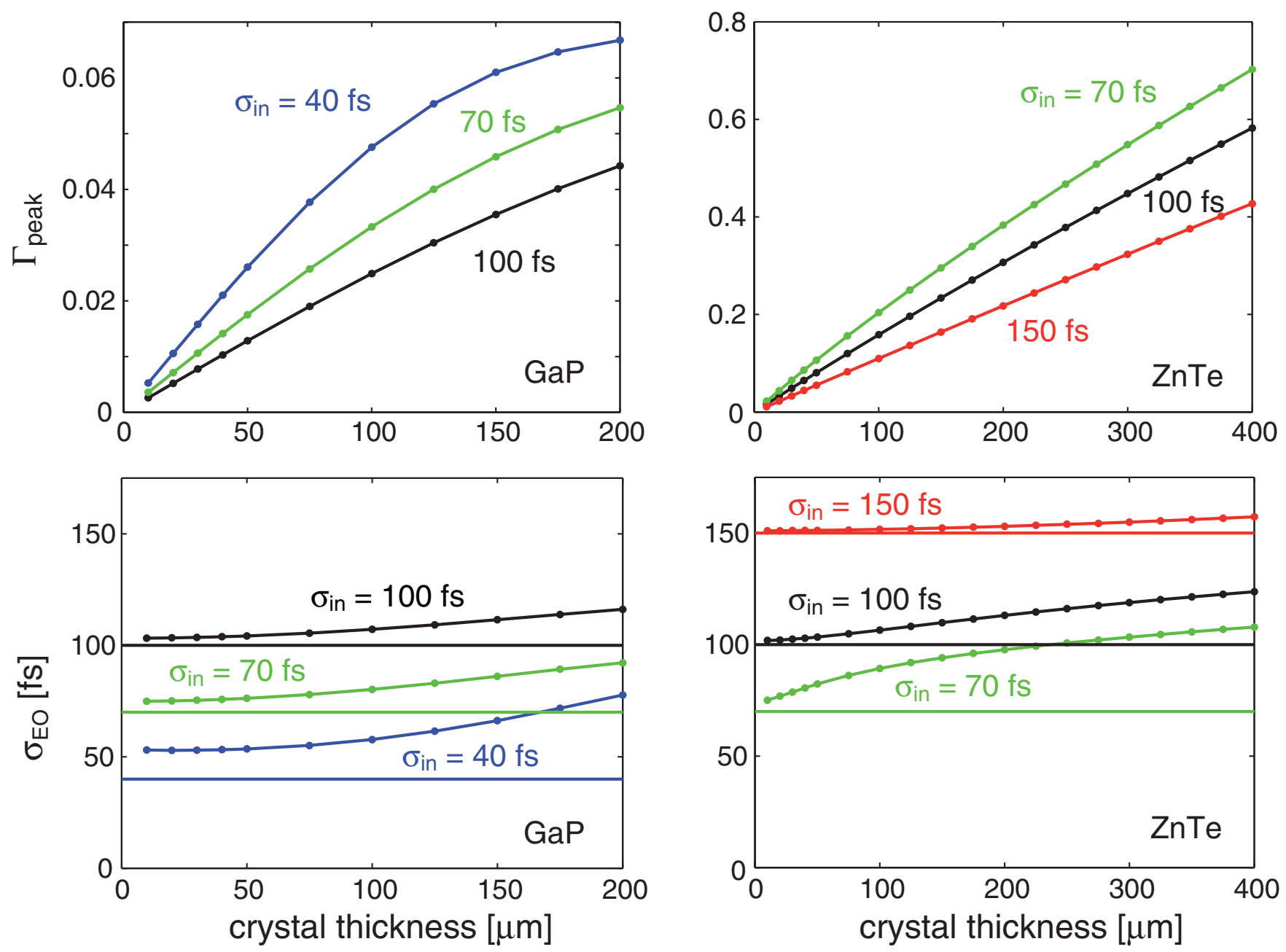

FIG. 22. (Color) Top graphs: Dependence of the electro-optic signal amplitude $\Gamma_{\text {peak }}$ on the thickness $d$ of the EO crystal for various initial pulse widths. Left: GaP, right: ZnTe. Bottom graphs: rms width $\sigma_{\mathrm{EO}}$ as a function of crystal thickness.

thickness of $1 \mathrm{~mm}$ or more may be chosen without much loss in time resolution.

\section{SUMMARY}

Using the available experimental data on the material properties of GaP and ZnTe (frequency-dependent complex refractive index and electro-optic coefficient), we have studied the effects of pulse broadening, pulse shape distortion, and phase and group velocity mismatch in the electro-optic detection of femtosecond electron bunches. Only the standard case has been considered where both the equivalent $\mathrm{THz}$ and the titanium-sapphire laser pulse impinge perpendicular to the surface of the EO crystal. Our conclusion is that the shortest rms pulse length which can be recovered without significant distortion amounts to $\sigma \approx$ $90 \mathrm{fs}$ in $\mathrm{ZnTe}$ and $\sigma \approx 50 \mathrm{fs}$ in GaP. The optimum thickness is 50 to $100 \mu \mathrm{m}$ for $\mathrm{GaP}$ and 100 to $300 \mu \mathrm{m}$ for $\mathrm{ZnTe}$.

We point out that any time jitter between the $\mathrm{THz}$ pulse and the laser pulse has been disregarded in our computations as well as experimental limitations such as detector resolution or the available laser-pulse length. We have thus restricted ourselves to an idealized electro-optic sampling (EOS) experiment in which the time profile of the electron bunches would have to be determined from measurements on many identical bunches. EO techniques permitting single-shot electron bunch diagnostics such as spectral, temporal, or spatial decoding have not been studied here. These single-shot techniques can at best achieve the time resolution derived in the present paper. In fact, in a recent single-shot EO experiment at FLASH [4], using a $65 \mu \mathrm{m}$ $\mathrm{GaP}$ crystal, the resolution limit has almost been reached.

[1] I. Wilke, A. M. MacLeod, W. A. Gillespie, G. Berden, G. M. H. Knippels, and A. F. G. van der Meer, Phys. Rev. Lett. 88, 124801 (2002).

[2] G. Berden, S. P. Jamison, A. M. McLeod, W. A. Gillespie, B. Redlich, and A. F. G. van der Meer, Phys. Rev. Lett. 93, 114802 (2004).

[3] A. L. Cavalieri et al., Phys. Rev. Lett. 94, 114801 (2005). 
[4] G. Berden, W. A. Gillespie, S. P. Jamison, E.-A. Knabbe, A. M. MacLeod, A. F. G. van der Meer, P. J. Phillips, H. Schlarb, B. Schmidt, P. Schmüser, and B. Steffen, Phys. Rev. Lett. 99, 164801 (2007).

[5] H. J. Bakker, G. C. Cho, H. Kurz, Q. Wu, and X.-C. Zhang, J. Opt. Soc. Am. B 15, 1795 (1998).

[6] M. Brunken et al., DESY Technical Report, TESLA Report No. 2003-11, 2003.

[7] A. Yariv and P. Yeh, Optical Waves in CrystalsPropagation and Control of Laser Radiation (John Wiley \& Sons, New York, 1984).

[8] N.C.J. van der Valk, T. Wenckebach, and P.C.M. Planken, J. Opt. Soc. Am. B 21, 622 (2004).

[9] P.C. M. Planken, H.-K.. Nienhuys, H.J. Bakker, and T. Wenckebach, J. Opt. Soc. Am. B 18, 313 (2001).

[10] K. Strössner, S. Ves, and M. Cardona, Phys. Rev. B 32, 6614 (1985).

[11] D. E. Aspnes and A. A. Studna, Phys. Rev. B 27, 985 (1983).

[12] D. T. Marple, J. Appl. Phys. 35, 539 (1964).

[13] L. Ward, Handbook of Optical Constants of Solids II (Academic Press, Boston, 1991), p. 737.

[14] A. N. Pikhtin, V. T. Prokopenko, and A. D. Yaskov, Sov. Phys. Semicond. 10, 1224 (1976).

[15] A. S. Barker, Jr., Phys. Rev. 136, A1290 (1964).

[16] G. Gallot, J. Zhang, R. W. McGowan, T.-I. Jeon, and D. Grischkowsky, Appl. Phys. Lett. 74, 3450 (1999).

[17] W. L. Faust and C. H. Henry, Phys. Rev. Lett. 17, 1265 (1966); W. L. Faust, C. H. Henry, and R. H. Eick, Phys. Rev. 173, 781 (1968).

[18] I. P. Kaminow, IEEE J. Quantum Electron. 4, 23 (1968).

[19] K. Tada and M. Aoki, Jpn. J. Appl. Phys. 10, 998 (1971).
[20] T. R. Sliker and J. M. Jost, J. Opt. Soc. Am. 56, 130 (1966).

[21] D. Berlincourt, H. Jaffe, and L. R. Shiozawa, Phys. Rev. 129, 1009 (1963).

[22] A. Leitenstorfer, S. Hunsche, J. Shah, M. C. Nuss, and W. H. Knox, Appl. Phys. Lett. 74, 1516 (1999).

[23] Q. Wu and X.-C. Zhang, Appl. Phys. Lett. 70, 1784 (1997).

[24] Gallot and Grischkowsky [25] chose the name "EO pulse" instead of "effective THz pulse."

[25] G. Gallot and D. Grischkowsky, J. Opt. Soc. Am. B 16, 1204 (1999).

[26] D. Meschede, Optics, Light and Lasers (Wiley-VCH, Weinheim, 2004).

[27] T. Kampfrath, J. Nötzold, and M. Wolf, Appl. Phys. Lett. 90, 231113 (2007).

[28] C. Kübler, R. Huber, S. Tübel, and A. Leitenstorfer, Appl. Phys. Lett. 85, 3360 (2004).

[29] We note that an extremely short electron bunch would be needed for generating a $10 \mathrm{fs} \mathrm{THz}$ pulse, but moreover, due to the $1 / \gamma$ opening angle of the relativistic Coulomb field, the Lorentz factor $\gamma$ would have to be larger than 1000 or the distance $r$ to the beam smaller than $5 \mathrm{~mm}$.

[30] D. A. Kleinman and W. Spitzer, Phys. Rev. 118, 110 (1960).

[31] T. Hattori, Y. Homma, A. Mitsuishi, and M. Tacke, Opt. Commun. 7, 229 (1973).

[32] M. Schall, M. Walther, and P. U. Jepsen, Phys. Rev. B 64, 094301 (2001).

[33] D. F. Nelson and E. H. Turner, J. Appl. Phys. 39, 3337 (1968). 